\title{
Enantioselective Total Synthesis of (+)-Gliocladin C
}

\author{
Larry E. Overman* and Youseung Shin \\ Department of Chemistry, 1102 Natural Sciences II, University of California, Irvine, California 92697-2025
}

Supporting Information

(34 pages)

\section{Table of Contents}
A. Experimental Procedures
$\mathrm{S} 1-\mathrm{S} 8$
B. Tabulated ${ }^{1} \mathrm{H}$ and ${ }^{13} \mathrm{C}$ NMR spectra of natural and synthetic (+)-gliocladin $\mathrm{C}$ S9-S10
C. Copies of ${ }^{1} \mathrm{H}$ and ${ }^{13} \mathrm{C}$ NMR spectra of new compounds
S11-S33
D. X-ray model of the $\mathrm{C} 3$ acetate analog of 13
S34

\section{A. Experimental Procedures}

General Details. Reactions were performed in oven-dried glassware fitted with rubber septa under an argon atmosphere. $\mathrm{CH}_{2} \mathrm{Cl}_{2}$ and THF were dried by passage through a bed of activated alumina. Commercial reagents were used without further purification. Thin-layer chromatography was performed on Merck $60 \mathrm{~F}_{254}$ precoated silica gel plate, which were visualized by exposure to UV $(254 \mathrm{~nm})$ or stained by submersion in $p$-anisaldehyde solution or ethanolic phosphomolybdic acid solution followed by heating on a hot plate. Flash column chromatography was performed in silica gel (230-400 mesh, Merck KGA). ${ }^{1} \mathrm{H}$ NMR spectra were recorded at 500 or $600 \mathrm{MHz}$ and ${ }^{13} \mathrm{C}$ NMR spectra at $125 \mathrm{MHz}$ or $150 \mathrm{MHz}$ with Brucker Avance spectrometers. Infrared spectra were recorded using an ASI ReactIR ${ }^{\mathrm{TM}} 1000$ spectrometer. Mass spectra were measured with a Micromass LCT spectrometer. Optical rotations were measured with a Jasco P-1010 polarimeter.

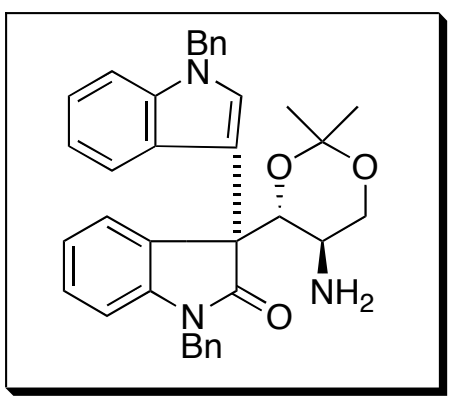

1,3-Dioxane 7. A methanol solution of $\mathrm{HCl}(3 \mathrm{M}, 30 \mathrm{~mL}$, prepared from $\mathrm{AcCl}$ and $\mathrm{MeOH}$ ) was added at room temperature to Mukaiyama aldol product $\mathbf{6}^{1}(2.57 \mathrm{~g}, 3.91$ mmol). After the reaction was completed (usually $1.5 \mathrm{~h}$, monitored by TLC), the solvent was removed on a rotary evaporator under reduced pressure keeping the bath temperature below $30{ }^{\circ} \mathrm{C}$ to suppress retroaldol reaction. Dichloromethane $(20 \mathrm{~mL})$ was added to the residue and this solution was concentrated under reduced pressure; this

\footnotetext{
${ }^{1}$ Adhikari, S.; Caille, S.; Hanbauer, M.; Ngo, V. X.; Overman, L. E. Org. Lett. 2005, 7, 2795-2798.
} 
procedure was repeated two times to remove all residual $\mathrm{HCl}-\mathrm{MeOH}$, which if present effects the next step. Diagnostic data for the amino diol intermedate: ${ }^{1} \mathrm{H}$ NMR $(500 \mathrm{MHz}$, $\left.\mathrm{CDCl}_{3}\right) \delta 7.90($ br s, $1 \mathrm{H}), 7.45(\mathrm{~m}, 1 \mathrm{H}), 7.41-7.28(\mathrm{~m}, 4 \mathrm{H}), 7.25-7.01(\mathrm{~m}, 10 \mathrm{H}), 6.92(\mathrm{~d}, J=$ $7.8 \mathrm{~Hz}, 1 \mathrm{H}), 6.86(\mathrm{~m}, 1 \mathrm{H}), 6.81(\mathrm{~m}, 1 \mathrm{H}), 6.76(\mathrm{br} \mathrm{d}, J=7.1 \mathrm{~Hz}, 1 \mathrm{H}), 6.51-5.74(\mathrm{br}, 2 \mathrm{H}), 5.37$ $(\mathrm{d}, J=3.7 \mathrm{~Hz}, 1 \mathrm{H}), 5.33(\mathrm{~m}, 1 \mathrm{H}), 5.14(\mathrm{~d}, J=15.5 \mathrm{~Hz}, 1 \mathrm{H}), 5.08$ (br s, 2H), 5.01 (d, $J=15.5$ $\mathrm{Hz}, 1 \mathrm{H}$ ), 4.97 (br s, 1H), 4.64 (br s, 1H), 3.84 (br s, 1H), 3.73 (br s, 1H).

A solution of this residue, 2,2-dimethoxypropane $(15 \mathrm{~mL})$, camphorsulfonic acid $(0.45 \mathrm{~g})$ and benzene $(15 \mathrm{~mL})$ was heated at $50{ }^{\circ} \mathrm{C}$ for $3 \mathrm{~h}$. After the reaction was completed, the reaction mixture was cooled to room temperature, and treated with saturated aqueous $\mathrm{NaHCO}_{3}(5 \mathrm{~mL})$. The phases were separated and the aqueous phase was extracted with EtOAc $(10 \mathrm{~mL})$. The combined organic layers were washed with brine, dried, and concentrated. Flash column chromatography $\left(\mathrm{MeOH}: \mathrm{CH}_{2} \mathrm{Cl}_{2}=1: 9\right)$ of the crude residue yielded $1.85 \mathrm{~g}(85 \%)$ of 7 as a colorless foam: IR (film) 3382, 3054, 2939, 1713, 1611, 1466, 1366, $1181 \mathrm{~cm}^{-1} ;{ }^{1} \mathrm{H}$ NMR $\left(500 \mathrm{MHz}, \mathrm{CDCl}_{3}\right) \delta 8.30-8.25(\mathrm{~m}, 1 \mathrm{H}), 7.53(\mathrm{~d}, J=7.3 \mathrm{~Hz}, 1 \mathrm{H})$, 7.32-7.14 (m, 12H), $7.10(\mathrm{dt}, J=1.0,7.5 \mathrm{~Hz}, 1 \mathrm{H}), 7.03-6.98(\mathrm{~m}, 2 \mathrm{H}), 6.92(\mathrm{~s}, 1 \mathrm{H}), 6.69(\mathrm{~d}, J$ $=7.3 \mathrm{~Hz}, 1 \mathrm{H}), 5.29(\mathrm{~d}, J=16.1 \mathrm{~Hz}, 1 \mathrm{H}), 5.23(\mathrm{~s}, 2 \mathrm{H}), 5.16(\mathrm{~d}, J=8.3 \mathrm{~Hz}, 1 \mathrm{H}), 4.48(\mathrm{~d}, J=$ $16.1 \mathrm{~Hz}, 1 \mathrm{H}), 3.81-3.73(\mathrm{~m}, 1 \mathrm{H}), 3.52-3.46(\mathrm{~m}, 2 \mathrm{H}), 1.57(\mathrm{~s}, 3 \mathrm{H}), 1.35-1.02(\mathrm{~s}, 2 \mathrm{H}), 1.18(\mathrm{~s}$, $3 \mathrm{H}) ;{ }^{13} \mathrm{C}$ NMR $\left(125 \mathrm{MHz}, \mathrm{CDCl}_{3}\right) \delta 177.2,144.1,137.8,137.3,136.0,128.9,128.5,127.9$, $127.7,127.4,127.2$, 126.6, 126.5, 125.9, 123.5, 122.7, 121.9, 120.2, 111.4, 110.5, 109.6, 99.5, 78.0, 65.9, 60.5, 56.9, 50.1, 48.5, 43.7, 28.1, 20.3; HRMS (CI) calcd for $\mathrm{C}_{36} \mathrm{H}_{35} \mathrm{O}_{3} \mathrm{~N}_{3}$ 557.2678, found 557.2657; $[\alpha]^{23}{ }_{\mathrm{D}}-122.8\left(c 5.0, \mathrm{CH}_{2} \mathrm{Cl}_{2}\right)$.

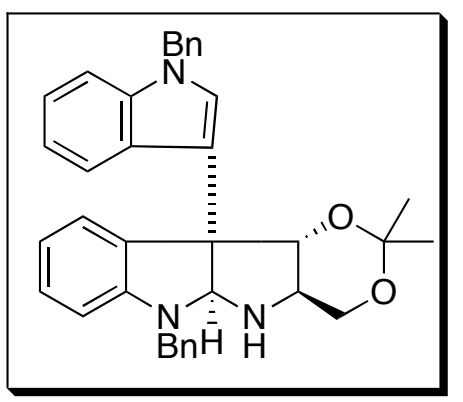

Pyrrolidinoindoline 8. A solution of aminodiol 7 (5.21 g, 9.35 $\mathrm{mmol})$ in THF $(20 \mathrm{~mL})$ was added dropwise to a stirring suspension of $\mathrm{LiAlH}_{4}(23.3 \mathrm{~mL}, 23.4 \mathrm{mmol}, 1.0 \mathrm{M}$ solution in $\mathrm{THF})$ in THF $(100 \mathrm{~mL})$ at room temperature. The reaction mixture was then stirred at room temperature for $2 \mathrm{~h}$, cooled to $0{ }^{\circ} \mathrm{C}$ and then carefully treated with $\mathrm{H}_{2} \mathrm{O}(50 \mathrm{~mL})$. The phases were separated and the aqueous phase was extracted with EtOAc. The combined organic extracts were washed with brine, dried and concentrated. The residue was dissolved in $\mathrm{MeOH}$ $(100 \mathrm{~mL})$ and silica gel $(10 \mathrm{~g})$ was added to the resultant solution. The mixture was stirred at room temperature open to the air for $4 \mathrm{~h}$, filtered, and the eluent was concentrated. Flash column chromatography (EtOAc : hexane $=1: 3$ ) of the crude product yielded $4.68 \mathrm{~g} \mathrm{(93 \% )}$ of $\mathbf{8}$ as a colorless oil: IR (film) 3350, 3051, 3031, 2877, 1605, 1488, 1355, 1173, $735 \mathrm{~cm}^{-1}$; ${ }^{1} \mathrm{H}$ NMR $\left(500 \mathrm{MHz}, \mathrm{CDCl}_{3}\right) \delta$ 7.82 (s, 1H), 7.44-7.40 (m, 2H), 7.32-7.22 (m, 6H), 7.16-7.12 $(\mathrm{m}, 3 \mathrm{H}), 7.09(\mathrm{dt}, J=7.8,1.3 \mathrm{~Hz}, 1 \mathrm{H}), 6.99(\mathrm{dt}, J=6.9,1.3 \mathrm{~Hz}, 1 \mathrm{H}), 6.87(\mathrm{dd}, J=7.3,0.9$ $\mathrm{Hz}, 1 \mathrm{H}), 6.73-6.65(\mathrm{~m}, 2 \mathrm{H}), 6.58(\mathrm{~d}, J=7.8 \mathrm{~Hz}, 1 \mathrm{H}), 6.52(\mathrm{t}, J=7.3 \mathrm{~Hz}, 1 \mathrm{H}), 5.41(\mathrm{~s}, 1 \mathrm{H})$, $5.23(\mathrm{~s}, 2 \mathrm{H}), 4.64(\mathrm{~d}, J=15.5 \mathrm{~Hz}, 1 \mathrm{H}), 4.41(\mathrm{~d}, J=15.5 \mathrm{~Hz}, 1 \mathrm{H}), 4.11-3.99(\mathrm{~m}, 2 \mathrm{H}), 3.79(\mathrm{t}$, $J=10.3 \mathrm{~Hz}, 1 \mathrm{H}), 3.57(\mathrm{dt}, J=10.3,4.6 \mathrm{~Hz}, 1 \mathrm{H}), 2.11(\mathrm{~s}, 1 \mathrm{H}), 1.51(\mathrm{~s}, 3 \mathrm{H}), 1.39(\mathrm{~s}, 3 \mathrm{H}) ;{ }^{13} \mathrm{C}$ NMR $\left(125 \mathrm{MHz}, \mathrm{CDCl}_{3}\right) \delta 148.6,138.4,137.7,136.7,133.4,129.1,128.9,128.6,128.4$, $128.0,127.9,127.7,127.3,124.5,121.7,120.6,119.2,117.5,113.7,109.9,106.3,100.8$, 87.9, 82.7, 68.2, 60.7, 56.4, 54.7, 50.5, 49.2, 29.9, 19.7; HRMS (CI) calcd for $\mathrm{C}_{36} \mathrm{H}_{35} \mathrm{O}_{2} \mathrm{~N}_{3}$ 541.2729, found 541.2725; $[\alpha]^{23}{ }_{\mathrm{D}}+137.6\left(c 3.0, \mathrm{CH}_{2} \mathrm{Cl}_{2}\right)$. 


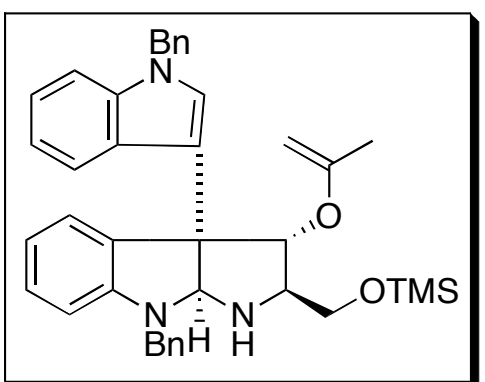

were washed with brine, dried, and concentrated. Flash column chromatography (EtOAc : hexane $=1: 15)$ of the crude product yielded $4.83 \mathrm{~g}(91 \%)$ of 9 as a colorless oil: IR (film) 3051, 2956, 1603, 1495, 1355, 1250, 1077, 872, $735 \mathrm{~cm}^{-1} ;{ }^{1} \mathrm{H}$ NMR (500 MHz, $\left.\mathrm{CDCl}_{3}\right) \delta$ $7.71(\mathrm{~d}, J=8.1 \mathrm{~Hz}, 1 \mathrm{H}), 7.34-7.18(\mathrm{~m}, 9 \mathrm{H}), 7.16-7.12(\mathrm{~m}, 1 \mathrm{H}), 7.09-6.98(\mathrm{~m}, 5 \mathrm{H}), 6.83(\mathrm{~s}$, $1 \mathrm{H}), 6.61(\mathrm{t}, J=7.5 \mathrm{~Hz}, 1 \mathrm{H}), 6.38(\mathrm{~d}, J=7.9 \mathrm{~Hz}, 1 \mathrm{H}), 5.46(\mathrm{~s}, 1 \mathrm{H}), 5.18(\mathrm{~s}, 2 \mathrm{H}), 5.10(\mathrm{~s}$, $1 \mathrm{H}), 4.50(\mathrm{~s}, 2 \mathrm{H}), 4.24(\mathrm{~s}, 1 \mathrm{H}), 3.83(\mathrm{~s}, 1 \mathrm{H}), 3.66(\mathrm{t}, J=7.8 \mathrm{~Hz}, 1 \mathrm{H}), 3.29-3.23(\mathrm{~m}, 1 \mathrm{H}), 2.94$ $(\mathrm{t}, J=9.1 \mathrm{~Hz}, 1 \mathrm{H}), 1.35(\mathrm{~s}, 3 \mathrm{H}) ;{ }^{13} \mathrm{C} \mathrm{NMR}\left(125 \mathrm{MHz}, \mathrm{CDCl}_{3}\right) \delta 159.0,149.3,139.1$, $138.1,137.5,133.4,129.0,128.9$, 128.8, 128.2, 127.8, 127.4, 127.0, 126.8, 125.1, 122.1, $121.7,119.1,117.9,117.0,109.9,107.2,88.9,85.2,83.3,67.2,65.4,60.8,50.2,49.2$, 21.4, 0.3; HRMS (CI) calcd for $\mathrm{C}_{39} \mathrm{H}_{44} \mathrm{~N}_{3} \mathrm{O}_{2} \mathrm{Si} 614.3203(\mathrm{M}+\mathrm{H})$, found $614.3187 ;[\alpha]^{23}{ }_{\mathrm{D}}+126.2(c$ 2.0, $\mathrm{CH}_{2} \mathrm{Cl}_{2}$ ).

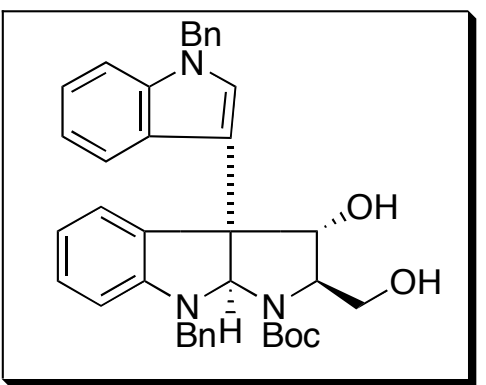

Diol 10. Aqueous $\mathrm{Na}_{2} \mathrm{CO}_{3}$ solution $(10 \%, 20 \mathrm{~mL})$ was added to a stirring solution of amine 9 (5.30 g, $8.64 \mathrm{mmol})$ in THF (20 $\mathrm{mL})$. After stirring at room temperature for $10 \mathrm{~min}, \mathrm{Boc}_{2} \mathrm{O}(1.89$ $\mathrm{g}, 8.67 \mathrm{mmol})$ was added. After $2 \mathrm{~h}, \mathrm{H}_{2} \mathrm{O}(50 \mathrm{~mL})$ and $\mathrm{Et}_{2} \mathrm{O}(50$ $\mathrm{mL})$ were added. The phases were separated and the aqueous phase was extracted twice with $\mathrm{Et}_{2} \mathrm{O}(20 \mathrm{~mL})$. The combined organic layers were washed with brine, dried, and concentrated. Flash column chromatography (EtOAc : hexane $=1: 5)$ of the crude residue yielded $6.20 \mathrm{~g}$ (quantitative yield) of the Boc derivative as a colorless oil: IR (film) 3029, 2991, 2931, 1696, 1604, 1467, 1374, $1216 \mathrm{~cm}^{-1}$; ${ }^{1} \mathrm{H}$ NMR $\left(500 \mathrm{MHz}, \mathrm{CDCl}_{3}\right) \delta 7.71(\mathrm{~d}, J=8.0 \mathrm{~Hz}, 1 \mathrm{H}), 7.38-7.22(\mathrm{~m}, 8 \mathrm{H}), 7.19-7.08(\mathrm{~m}$, 2H), 7.09-7.03 (m, 4H), 6.83-6.68 (m, 2H), 6.32-6.19 (m, 2H), $5.45(\mathrm{~s}, 1 \mathrm{H}), 5.21(\mathrm{~s}, 2 \mathrm{H})$, $5.15(\mathrm{~s}, 1 \mathrm{H}), 4.51(\mathrm{~s}, 2 \mathrm{H}), 4.29(\mathrm{~s}, 1 \mathrm{H}), 3.87(\mathrm{~s}, 1 \mathrm{H}), 3.75-3.67(\mathrm{~m}, 1 \mathrm{H}), 3.25-3.21(\mathrm{~m}, 1 \mathrm{H})$, $2.93(\mathrm{t}, J=9.8 \mathrm{~Hz}, 1 \mathrm{H}), 1.43(\mathrm{~s}, 9 \mathrm{H}), 1.35(\mathrm{~s}, 3 \mathrm{H}), 0.01(\mathrm{~s}, 9 \mathrm{H}) ;{ }^{13} \mathrm{C} \mathrm{NMR}\left(125 \mathrm{MHz}, \mathrm{CDCl}_{3}\right)$ $\delta 172.3,159.1,149.4,139.0,138.1,137.7,133.2,129.1,128.8,128.7,128.5,128.4,128.1$,

1. $\quad{ }^{2}$ Rychnovsky, S. D.; Kim, J. Tetrahedron Lett. 1991, 32, 7219. 
127.9, 127.5, 126.9, 125.1, 122.3, 119.0, 117.8, 117.2, 109.9, 107.5, 88.7, 85.3, 83.5, 67.1, 65.4, 60.9, 50.5, 49.1, 28.6, 24.7, 21.3, 0.1; HRMS (CI) calcd for $\mathrm{C}_{44} \mathrm{H}_{52} \mathrm{~N}_{3} \mathrm{O}_{4} \mathrm{Si} 714.3728(\mathrm{M}$ $+\mathrm{H})$, found $714.3731 ;[\alpha]^{26}+46.8\left(c 0.26, \mathrm{CHCl}_{3}\right)$.

Oxalic acid $(0.22 \mathrm{~g}, 1.75 \mathrm{mmol})$ was added to a stirred solution of this Bocpyrrolidinoindoline intermediate $(6.16 \mathrm{~g}, 8.64 \mathrm{mmol})$ in $\mathrm{MeOH}(48 \mathrm{~mL})$ at room temperature. After stirring for $1 \mathrm{~h}$ at room temperature, saturated aqueous $\mathrm{NaHCO}_{3}(10 \mathrm{~mL})$ and EtOAc $(50 \mathrm{~mL})$ were added. The phases were separated and the aqueous phase was extracted twice with EtOAc $(20 \mathrm{~mL})$. The combined organic layers were washed with brine, dried, and concentrated. Flash column chromatography (EtOAc : hexane $=1: 2$ ) of the crude material yielded $3.68 \mathrm{~g}$ (71\%) of diol $\mathbf{1 0}$ as a colorless oil: IR (film) 3423, 3028, 2993, 2929, 1697, 1605, 1466, 1374, $1217 \mathrm{~cm}^{-1}$; ${ }^{1} \mathrm{H}$ NMR $\left(500 \mathrm{MHz}, \mathrm{CDCl}_{3}\right)$ \& 7.82-7.66 (m, $\left.1 \mathrm{H}\right), 7.35-7.12$ $(\mathrm{m}, 12 \mathrm{H}), 7.12-7.03(\mathrm{~m}, 3 \mathrm{H}), 6.74-6.62(\mathrm{~m}, 1 \mathrm{H}), 6.42-6.28(\mathrm{~m}, 1 \mathrm{H}), 6.15(\mathrm{~s}, 1 \mathrm{H}), 5.14(\mathrm{~s}$, 2H), $5.03(\mathrm{~s}, 1 \mathrm{H}), 4.93(\mathrm{t}, J=16.0 \mathrm{~Hz}, 1 \mathrm{H}), 4.67(\mathrm{~s}, 2 \mathrm{H}), 4.44-4.21(\mathrm{~m}, 1 \mathrm{H}), 3.87-3.64(\mathrm{~m}$, $1 \mathrm{H}), 3.11-2.89(\mathrm{~m}, 1 \mathrm{H}), 2.37$ (br s, 1H), $1.83(\mathrm{br} \mathrm{s}, 1 \mathrm{H}), 1.43(\mathrm{~s}, 9 \mathrm{H}) ;{ }^{13} \mathrm{C}$ NMR $(125 \mathrm{MHz}$, $\left.\mathrm{CDCl}_{3}\right) \delta$ 172.3, 159.1, 149.4, 139.0, 138.1, 137.7, 133.2, 129.1, 128.8, 128.7, 128.5, 128.4, 128.1, 127.9, 127.5, 126.9, 125.1, 122.3, 119.0, 117.8, 117.2, 109.9, 88.7, 83.5, 67.1, 65.4, 60.9, 50.5, 49.1, 28.6, 24.7; HRMS (CI) calcd for $\mathrm{C}_{38} \mathrm{H}_{40} \mathrm{~N}_{3} \mathrm{O}_{4} 602.3020(\mathrm{M}+\mathrm{H})$, found 602.3024; $[\alpha]_{\mathrm{D}}^{25}+109.7\left(c 0.75, \mathrm{CHCl}_{3}\right)$.

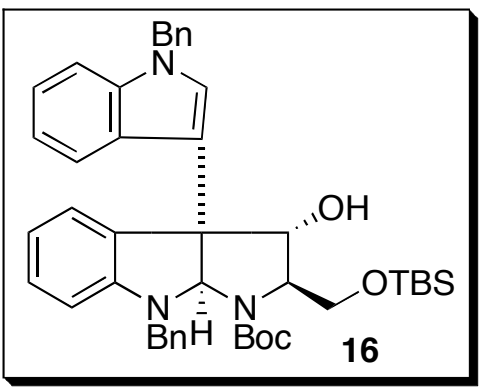

TBS Intermediate 16. NaH (60\% dispersion in mineral oil, $0.49 \mathrm{~g}, 12.2 \mathrm{mmol})$ was added to a stirring solution of diol $\mathbf{1 0}$ $(3.68 \mathrm{~g}, 6.11 \mathrm{mmol})$ in THF $(100 \mathrm{~mL})$ at room temperature. After stirring for $10 \mathrm{~min}, \mathrm{TBSCl}(1.0 \mathrm{~g}, 6.63 \mathrm{mmol})$ was added and the reaction mixture was stirred for an additional $2 \mathrm{~h}$ at room temperature. Brine $(50 \mathrm{~mL})$ and $\mathrm{Et}_{2} \mathrm{O}(100 \mathrm{~mL})$ were added, the phases were separated, and the aqueous phase was extracted twice with $\mathrm{Et}_{2} \mathrm{O}$. The combined organic layers were dried and concentrated. Flash column chromatography (EtOAc : hexane $=1: 9$ ) of the residue yielded $4.20 \mathrm{~g}(96 \%)$ of $\mathbf{1 6}$ as a colorless oil: IR (film) 3489, 3030, 2957, 2930, 1695, 1606, 1467, 1366, $1254 \mathrm{~cm}^{-1} ;{ }^{1} \mathrm{H}$ NMR $\left(500 \mathrm{MHz}, \mathrm{CDCl}_{3}\right) \delta 7.82-$ $7.67(\mathrm{~m}, 1 \mathrm{H}), 7.36-7.11(\mathrm{~m}, 12 \mathrm{H}), 7.12-7.04(\mathrm{~m}, 3 \mathrm{H}), 6.76-6.62(\mathrm{~m}, 1 \mathrm{H}), 6.42-6.28(\mathrm{~m}, 1 \mathrm{H})$, $6.17(\mathrm{~s}, 1 \mathrm{H}), 5.16(\mathrm{~s}, 2 \mathrm{H}), 5.02(\mathrm{~s}, 1 \mathrm{H}), 4.92(\mathrm{t}, J=15.6 \mathrm{~Hz}, 1 \mathrm{H}), 4.65(\mathrm{~s}, 2 \mathrm{H}), 4.47-4.21(\mathrm{~m}$, $1 \mathrm{H}), 3.88-3.64(\mathrm{~m}, 1 \mathrm{H}), 3.11-2.87(\mathrm{~m}, 1 \mathrm{H}), 2.34(\mathrm{br} \mathrm{s}, 1 \mathrm{H}), 1.43(\mathrm{~s}, 9 \mathrm{H}), 0.86(\mathrm{~s}, 9 \mathrm{H}),-0.03$ $(\mathrm{s}, 6 \mathrm{H}) ;{ }^{13} \mathrm{C}$ NMR $\left(125 \mathrm{MHz}, \mathrm{CDCl}_{3}\right) \delta 172.1,159.2,149.7,139.2,138.0,137.7,133.4$, $129.2,128.8,128.7,128.5,128.4,128.0,127.8,127.5,126.7,125.1,122.5,119.1,117.9$, 117.2, 109.8, 88.6, 83.5, 67.2, 65.6, 60.9, 50.7, 49.3, 28.8, 24.6, 14.5, -3.2, -4.9; HRMS (CI) calcd for $\mathrm{C}_{44} \mathrm{H}_{54} \mathrm{~N}_{3} \mathrm{O}_{4} \mathrm{Si} 716.3884(\mathrm{M}+\mathrm{H})$, found 716.3891; $[\alpha]^{25}{ }_{\mathrm{D}}+99.8\left(c 1.2, \mathrm{CHCl}_{3}\right)$.

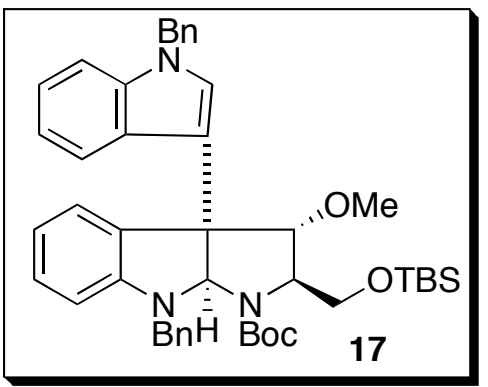

Methyl TBS Intermediate 17. $\mathrm{NaH}(60 \%$ dispersion in mineral oil, $36 \mathrm{mg}, 0.92 \mathrm{mmol})$ was added to a stirring solution of alcohol $16(101 \mathrm{mg}, 0.14 \mathrm{mmol})$ in THF $(2.8 \mathrm{~mL})$ at $0{ }^{\circ} \mathrm{C}$. After $10 \mathrm{~min}$, MeI $(0.088 \mathrm{~mL}, 1.4 \mathrm{mmol})$ was added dropwise and the reaction mixture was warmed to room temperature. After $2 \mathrm{~h}$, the reaction mixture was quenched by adding $\mathrm{H}_{2} \mathrm{O}(10 \mathrm{~mL})$ and $\mathrm{Et}_{2} \mathrm{O}(10 \mathrm{~mL})$ was added. The phases were separated and the aqueous phase was extracted 
twice with $\mathrm{Et}_{2} \mathrm{O}(5 \mathrm{~mL})$. The combined organic layers were washed with brine, dried and concentrated. Flash column chromatography (EtOAc : hexane $=1: 9$ ) of the crude material yielded $79.2 \mathrm{mg}$ (77\%) of $\mathbf{1 7}$ as a colorless oil: IR (film) 2954, 2929, 2856, 1694, 1603, 1495, 1453, 1389, 1250, 1158, 1096, $835 \mathrm{~cm}^{-1}$; ${ }^{1} \mathrm{H}$ NMR $\left(500 \mathrm{MHz}, \mathrm{CDCl}_{3}\right) \delta 7.71$ (d, $J=7.9$ $\mathrm{Hz}, 1 \mathrm{H}), 7.39-7.17(\mathrm{~m}, 8 \mathrm{H}), 7.15-7.10(\mathrm{~m}, 2 \mathrm{H}), 6.99$ (s, 1H), 6.77 (m, 3H), 6.31 (d, J = 7.7 $\mathrm{Hz}, 1 \mathrm{H}), 6.17(\mathrm{~s}, 1 \mathrm{H}), 5.35-5.20(\mathrm{~m}, 3 \mathrm{H}), 4.75-4.68(\mathrm{~m}, 3 \mathrm{H}), 4.55(\mathrm{~m}, 1 \mathrm{H}), 3.79(\mathrm{~m}, 1 \mathrm{H})$, $3.30(\mathrm{~s}, 3 \mathrm{H}), 3.03(\mathrm{~m}, 1 \mathrm{H}), 1.52-1.39(\mathrm{~m}, 9 \mathrm{H}), 0.95-0.93(\mathrm{~m}, 9 \mathrm{H}), 0.05-0.01(\mathrm{~m}, 6 \mathrm{H}) ;{ }^{13} \mathrm{C}$ NMR $\left(125 \mathrm{MHz}, \mathrm{CDCl}_{3}\right) \delta 154.5,148.7,139.2,137.7,137.5,131.9,128.8,128.5,128.3$, $127.7,127.5,127.3,126.9,126.6,126.0,126.3,124.8,122.0,121.6,119.1,117.8,115.6$, $109.9,107.2,88.5,87.8,80.7,66.2,62.4,60.6,57.7,50.1,29.8,28.5,28.3,25.9,-5.4$; HRMS (CI) calcd for $\mathrm{C}_{45} \mathrm{H}_{55} \mathrm{~N}_{3} \mathrm{O}_{4} \mathrm{Si} 752.3860(\mathrm{M}+\mathrm{Na})$, found 752.3864; $[\alpha]^{24}+36.4(c 0.11$, $\left.\mathrm{CHCl}_{3}\right)$.

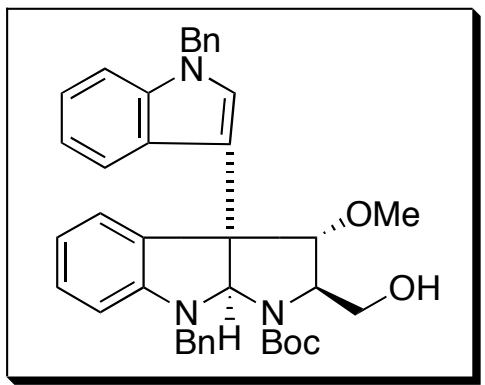

Alcohol Intermediate 11. TBAF (1.0 M solution in THF, $0.11 \mathrm{~mL}$ ) was added dropwise to a stirred solution of TBSprotected alcohol $17(79.2 \mathrm{mg}, 0.11 \mathrm{mmol})$ in THF $(5 \mathrm{~mL})$ at room temperature. After $12 \mathrm{~h}$ at room temperature, the reaction was concentrated and the residue was purified by flash chromatography (EtOAc : hexane $=3: 7$ ) to yield 61.2 mg (92\%) of alcohol $\mathbf{1 1}$ as a colorless foam: IR (film) 2981, 2954, 1677, 1603, 1391, 1368, 1266, 1177, 1138, 1096, 908, $735 \mathrm{~cm}^{-1} ;{ }^{1} \mathrm{H}$ NMR $\left(500 \mathrm{MHz}, \mathrm{CDCl}_{3}\right) \delta 7.46(\mathrm{~s}, 1 \mathrm{H}), 7.38-$ $7.28(\mathrm{~m}, 9 \mathrm{H}), 7.22(\mathrm{~m}, 2 \mathrm{H}), 7.15(\mathrm{~m}, 3 \mathrm{H}), 7.08(\mathrm{~m}, 1 \mathrm{H}), 6.77(\mathrm{t}, J=7.3 \mathrm{~Hz}, 1 \mathrm{H}), 6.44(\mathrm{~m}$, $1 \mathrm{H}), 6.15(\mathrm{~m}, 1 \mathrm{H}), 5.37(\mathrm{~m}, 2 \mathrm{H}), 4.80-4.63(\mathrm{~m}, 2 \mathrm{H}), 4.57(\mathrm{~m}, 1 \mathrm{H}), 4.28(\mathrm{~m}, 1 \mathrm{H}), 3.83-3.60$ $(\mathrm{m}, 3 \mathrm{H}), 3.41(\mathrm{~s}, 3 \mathrm{H}), 1.48-1.32(\mathrm{~m}, 9 \mathrm{H}) ;{ }^{13} \mathrm{C} \mathrm{NMR}\left(125 \mathrm{MHz}, \mathrm{CDCl}_{3}\right) \delta 156.5,148.9,138.9$, $137.7,137.1,128.8,128.7,128.3,127.6,126.9,126.6,124.8,121.7,121.5,119.2,118.2$, $112.6,110.0,108.0,90.5,89.8,81.7,67.3,65.7,58.8,51.0,50.2,28.1,21.1$; HRMS (CI) calcd for $\mathrm{C}_{39} \mathrm{H}_{41} \mathrm{~N}_{3} \mathrm{O}_{4} \mathrm{Na} 638.2995(\mathrm{M}+\mathrm{Na})$, found 638.2996; $[\alpha]^{24}+57.4\left(c 0.2, \mathrm{CHCl}_{3}\right)$.

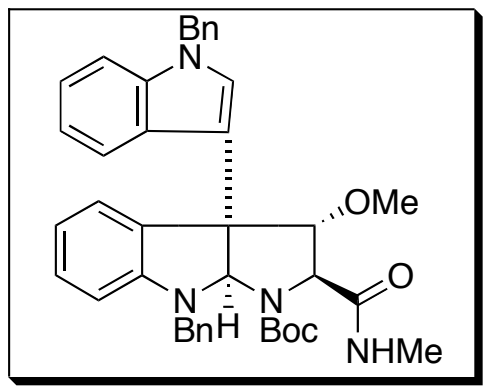

Boc-Protected Carboxamide 12. Dess-Martin periodinane ${ }^{3}$ $(0.25 \mathrm{~g}, 0.60 \mathrm{mmol})$ and pyridine $(0.097 \mathrm{~mL})$ were added to a solution of alcohol $11(61.2 \mathrm{mg}, 0.10 \mathrm{mmol})$ and $\mathrm{CH}_{2} \mathrm{Cl}_{2}(2$ $\mathrm{mL})$ at room temperature. After $1 \mathrm{~h}$, the mixture was quenched with saturated aqueous $\mathrm{NaHCO}_{3}(10 \mathrm{~mL})$ and saturated aqueous $\mathrm{Na}_{2} \mathrm{~S}_{2} \mathrm{O}_{3}(10 \mathrm{~mL})$. The resulting mixture was extracted with ethyl ether $(10 \mathrm{~mL} \times 2)$ and the combined extracts were dried over anhydrous $\mathrm{MgSO}_{4}$. Filtration and concentration provided the crude aldehyde intermediate as a pale yellow oil, which was used immediately in the next reaction without further purification.

Following the general procedure of Pinnick, ${ }^{4}$ a $2 \mathrm{M}$ solution of 2-methyl-2-butene in THF (0.28 mL, $0.57 \mathrm{mmol}), \mathrm{NaH}_{2} \mathrm{PO}_{4} \cdot \mathrm{H}_{2} \mathrm{O}(0.041 \mathrm{~g}, 0.30 \mathrm{mmol})$, and $\mathrm{NaClO}_{2}(0.24 \mathrm{~g}, 0.30$ $\mathrm{mmol}$ ) were added successively at room temperature to a stirring mixture of this crude aldehyde, THF $(0.5 \mathrm{~mL})$ and $\mathrm{H}_{2} \mathrm{O}(0.5 \mathrm{~mL})$. The reaction mixture was then stirred for an

\footnotetext{
3 (a) Dess, D. B.; Martin, J. C. J. Org. Chem. 1983, 48, 4155-4156. (b) Meyer, S. D.; Schreiber, S. L. J. Org. Chem. 1994, 59, 7549-7552.

${ }^{4}$ Bal, B. S.; Childers, W. E.; Pinnick, H. W. Tetrahedron, 1981, 37, 2091.
} 
additional $2 \mathrm{~h}$ at room temperature, diluted with $\mathrm{H}_{2} \mathrm{O}(10 \mathrm{~mL})$, and extracted with EtOAc $(2 \mathrm{x}$ $10 \mathrm{~mL}$ ). The combined organic layers were dried over $\mathrm{MgSO}_{4}$, concentrated, and the residual crude acid was used for the next reaction without further purification.

Benzotriazole-1-yloxy-tris(dimethylamino)phosphonium hexafluorophosphate (BOP, 48 $\mathrm{mg}, 0.11 \mathrm{mmol}), \mathrm{MeNH}_{3} \mathrm{Cl}(7.4 \mathrm{mg}, 0.11 \mathrm{mmol})$, and $\mathrm{Et}_{3} \mathrm{~N}(0.021 \mathrm{~mL}, 0.15 \mathrm{mmol})$ were added successively at room temperature to a stirring solution of the crude acid in $\mathrm{CH}_{2} \mathrm{Cl}_{2}$ (1 $\mathrm{mL}$ ). After $3 \mathrm{~h}$, the reaction was completed and the reaction was concentrated. The residue was purified by flash chromatography (hexane : EtOAc $=3: 2$ ) to yield $38.1 \mathrm{mg}(60 \%$ for three steps) of amide product 12 as a pale yellow oil: IR (film) 3054, 2987, 1673, 1441, 1370,1266, 1144, $895 \mathrm{~cm}^{-1}$; ${ }^{1} \mathrm{H}$ NMR $\left(500 \mathrm{MHz}, \mathrm{CDCl}_{3}\right) \delta 7.92(\mathrm{~d}, J=7.9 \mathrm{~Hz}, 1 \mathrm{H}), 7.49-$ 7.42 (m, 4H), 7.34-7.29 (m, 3H), 7.28-7.23 (m, 3H), 7.19-7.14 (m, 2H), 7.09-7.07 (m, 3H), $6.73(\mathrm{~m}, 1 \mathrm{H}), 6.22(\mathrm{~s}, 1 \mathrm{H}), 5.87(\mathrm{br} \mathrm{s}, 1 \mathrm{H}), 5.24(\mathrm{~d}, J=16.4 \mathrm{~Hz}, 1 \mathrm{H}), 5.21(\mathrm{~d}, J=16.3 \mathrm{~Hz}$, $1 \mathrm{H}), 4.91(\mathrm{~m}, 1 \mathrm{H}), 4.70(\mathrm{~m}, 2 \mathrm{H}), 3.41(\mathrm{~m}, 3 \mathrm{H}), 3.09(\mathrm{~d}, J=5.0 \mathrm{~Hz}, 3 \mathrm{H}), 2.37(\mathrm{~d}, J=4.9 \mathrm{~Hz}$, 3H), 1.49 (m, 9H); ${ }^{13} \mathrm{C}$ NMR (125 MHz, $\mathrm{CDCl}_{3}$ ) $\delta$ 170.2, 155.4, 148.7, 142.4, 139.9, 137.5, 131.2, 129.0, 128.7, 128.6, 128.33, 128.25, 127.5, 127.2, 126.6, 121.7, 119.3, 118.7, 110.0, 92.5, 68.2, 51.1, 50.1, 29.8, 28.3, 26.9, 26.0 ; HRMS (CI) calcd for $\mathrm{C}_{40} \mathrm{H}_{42} \mathrm{~N}_{4} \mathrm{O}_{4} \mathrm{Na} 665.3104$ $(\mathrm{M}+\mathrm{Na})$, found $665.3103 ;[\alpha]^{25}+52.1\left(c 0.25, \mathrm{CHCl}_{3}\right)$.

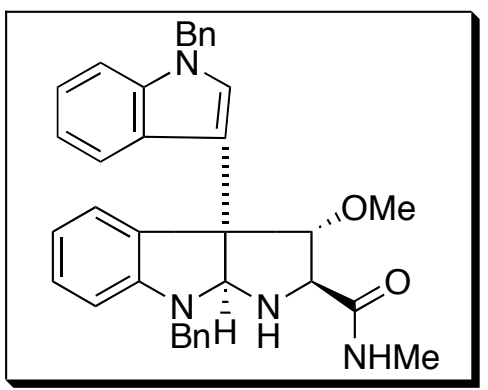

Aminocarboxamide 13. Following the general procedure Danishefsky, ${ }^{5}$ Boc-protected amide 12 (49.8 mg, $\left.77.5 \mu \mathrm{mol}\right)$ was dissolved in dry $\mathrm{MeCN}(1.5 \mathrm{~mL})$ and this solution was cooled to $0{ }^{\circ} \mathrm{C}$ under argon. TMSI $(0.066 \mathrm{~mL}, 0.47 \mathrm{mmol})$ was then added dropwise over $10 \mathrm{~min}$. After $30 \mathrm{~min}$, the reaction mixture was poured into sat. aqueous $\mathrm{NaHCO}_{3}(20$ $\mathrm{mL})$ and extracted with EtOAc $(2 \times 10 \mathrm{~mL})$. The combined organic layers were washed with brine, dried, filtered, and concentrated. Flash column chromatography (EtOAc : hexane $=1: 1)$ of the crude residue yielded $27.3 \mathrm{mg}(65 \%)$ of aminocarboxamide $\mathbf{1 3}$ as a colorless oil: IR (film) 3369, 2925, 1733, 1665, 1603, 1530, 1482, 1453, 1353, 1245, $1092 \mathrm{~cm}^{-1} ;{ }^{1} \mathrm{H}$ NMR $\left(500 \mathrm{MHz}, \mathrm{CDCl}_{3}\right) \delta 7.74(\mathrm{~d}, J=8.0 \mathrm{~Hz}, 1 \mathrm{H}), 7.42-7.39(\mathrm{~m}, 2 \mathrm{H}), 7.37-7.31(\mathrm{~m}, 7 \mathrm{H})$, $7.27(\mathrm{~d}, J=8.2 \mathrm{~Hz}, 1 \mathrm{H}), 7.21-7.16(\mathrm{~m}, 3 \mathrm{H}), 7.13-7.10(\mathrm{~m}, 1 \mathrm{H}), 7.08(\mathrm{dt}, J=1.1,7.6 \mathrm{~Hz}, 1 \mathrm{H})$, $6.94(\mathrm{~m}, 1 \mathrm{H}), 6.69(\mathrm{t}, J=7.5 \mathrm{~Hz}, 1 \mathrm{H}), 6.49(\mathrm{~d}, J=7.8 \mathrm{~Hz}, 1 \mathrm{H}), 5.42(\mathrm{~s}, 1 \mathrm{H}), 5.26(\mathrm{~s}, 2 \mathrm{H})$, $4.94(\mathrm{~d}, J=2.0 \mathrm{~Hz}, 1 \mathrm{H}), 4.60(\mathrm{~d}, J=14.7 \mathrm{~Hz}, 1 \mathrm{H}), 4.48(\mathrm{~d}, J=14.6 \mathrm{~Hz}, 1 \mathrm{H}), 4.08(\mathrm{~s}, 1 \mathrm{H})$, $3.44(\mathrm{~s}, 3 \mathrm{H}), 2.39(\mathrm{~d}, J=5.0 \mathrm{~Hz}, 3 \mathrm{H}) ;{ }^{13} \mathrm{C}$ NMR $\left(125 \mathrm{MHz}, \mathrm{CDCl}_{3}\right) \delta 172.6,148.8,138.1$, 137.4, 137.2, 132.4, 128.77, 128.75, 128.67, 128.0, 127.6, 127.4, 127.1, 126.3, 125.0, 121.6, 121.0, 119.1, 118.5, 114.6, 109.9, 107.2, 90.7, 89.0, 67.6, 59.7, 58.1, 50.1, 49.9, 29.8, 25.5; HRMS (CI) calcd for $\mathrm{C}_{35} \mathrm{H}_{34} \mathrm{O}_{2} \mathrm{~N}_{4} \mathrm{Na} 565.2579$, found 565.2580; $[\alpha]^{26}{ }_{\mathrm{D}}+66.3\left(c 0.10, \mathrm{CHCl}_{3}\right)$.

5 Depew, K. M.; Marsden, S.P.; Zatorska, D.; Zatorski, A.; Bornmann, W. G.; Danishefsky, S. J. J. Am. Chem. Soc. 1999, $121,11953$. 


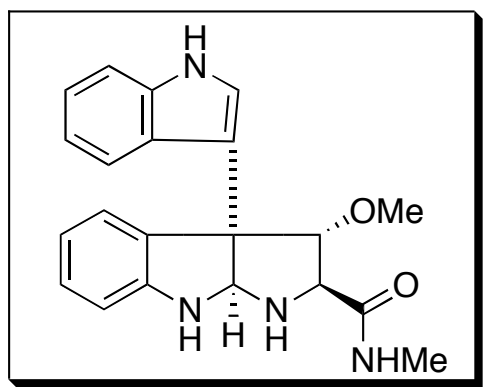

by replacing the cooling bath with a water bath. The residue was dissolved in $\mathrm{CH}_{2} \mathrm{Cl}_{2}(4 \mathrm{~mL})$ and filtered through a cotton-plugged pippet. After concentration of the filtrate, flash column chromatography (MeOH: $\left.\mathrm{CH}_{2} \mathrm{Cl}_{2}=1: 9\right)$ of the crude residue yielded $10.0 \mathrm{mg}(87 \%)$ of amine 14 as a colorless oil: IR (film) 3330, 2927, 1648, 1605,1538, 1484, 1461, 1410, 1241,1100, 1081, $1025 \mathrm{~cm}^{-1} ;{ }^{1} \mathrm{H}$ NMR $\left(500 \mathrm{MHz}, \mathrm{CDCl}_{3}\right) \delta 8.07$ (br s, 1H), 7.85 (d, J= 7.9 $\mathrm{Hz}, 1 \mathrm{H}), 7.30(\mathrm{dd}, J=10.4,8.1 \mathrm{~Hz}, 1 \mathrm{H}), 7.16-7.12(\mathrm{~m}, 3 \mathrm{H}), 7.17(\mathrm{t}, J=7.8 \mathrm{~Hz}, 1 \mathrm{H}), 7.13-$ $7.10(\mathrm{~m}, 2 \mathrm{H}), 7.02(\mathrm{dt}, J=1.0,7.6 \mathrm{~Hz}, 1 \mathrm{H}), 6.98(\mathrm{~d}, J=2.4 \mathrm{~Hz}, 1 \mathrm{H}), 6.70(\mathrm{t}, J=7.5 \mathrm{~Hz}, 1 \mathrm{H})$, $6.64(\mathrm{~d}, J=7.8 \mathrm{~Hz}, 1 \mathrm{H}), 5.62(\mathrm{~s}, 1 \mathrm{H}), 4.89(\mathrm{~s}, 1 \mathrm{H}), 4.02(\mathrm{~s}, 1 \mathrm{H}), 3.37(\mathrm{~s}, 3 \mathrm{H}), 2.32(\mathrm{~d}, J=5.0$ $\mathrm{Hz}, 3 \mathrm{H}) ;{ }^{13} \mathrm{C}$ NMR $\left(125 \mathrm{MHz}, \mathrm{CDCl}_{3}\right) \delta 172.4,148.0,137.0,131.4,128.8,126.5,125.4$, $122.5,121.8,121.1,119.6,119.4,115.9,111.4,109.8,90.7,84.5,68.3,61.4,57.8,25.4$; HRMS (CI) calcd for $\mathrm{C}_{21} \mathrm{H}_{22} \mathrm{O}_{2} \mathrm{~N}_{4} \mathrm{Na} 385.1640$, found 385.1641; $[\alpha]^{23}{ }_{\mathrm{D}}+23.5\left(c 0.19, \mathrm{CHCl}_{3}\right)$.

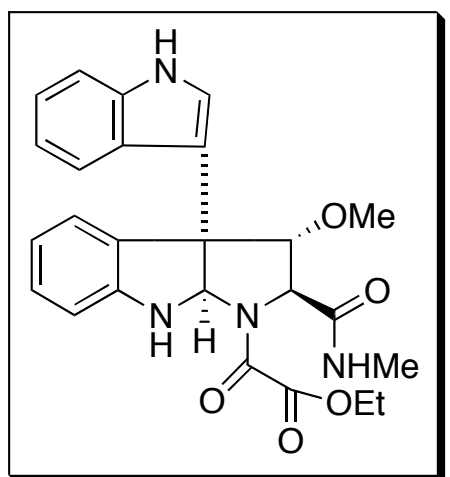

Oxalyl Amide 15. Ethyl chlorooxoacetate $(5.0 \mu \mathrm{L}, 44 \mu \mathrm{mol})$ and $\mathrm{Et}_{3} \mathrm{~N}(6 \mu \mathrm{L}, 44 \mu \mathrm{mol})$ were added at room temperature to a solution of triamine amide $14(10.7 \mathrm{mg}, 30.0 \mu \mathrm{mol})$ in dry $\mathrm{CH}_{2} \mathrm{Cl}_{2}(0.6 \mathrm{~mL})$. After $30 \mathrm{~min}$, the solvent was evaporated under reduced pressure, and the resulting residue was subjected to a flash column chromatography (EtOAc : hexane $=7: 3$ ) to give $11.8 \mathrm{mg}(87 \%)$ of $\mathbf{1 5}$ as a colorless oil: IR (film) 3357, 2927, 1737, 1656, 1542, 1466, 1414, 1250, 1218, 1162, 1102, $1015 \mathrm{~cm}^{-1} ;{ }^{1} \mathrm{H}$ NMR $\left(500 \mathrm{MHz}, \mathrm{CDCl}_{3}\right) \delta 8.07$ (br s, 1H), 7.71 $(\mathrm{d}, J=8.1 \mathrm{~Hz}, 1 \mathrm{H}), 7.33(\mathrm{~d}, J=7.7 \mathrm{~Hz}, 2 \mathrm{H}), 7.19(\mathrm{~m}, 1 \mathrm{H})$, 7.15-7.07 (m, 2H), $7.03(\mathrm{~s}, 1 \mathrm{H}), 6.79-6.76(\mathrm{~m}, 1 \mathrm{H}), 6.68(\mathrm{~d}, J=$ $7.6 \mathrm{~Hz}, 1 \mathrm{H}), 6.14(\mathrm{~s}, 1 \mathrm{H}), 5.55(\mathrm{~s}, 1 \mathrm{H}), 5.03(\mathrm{~s}, 1 \mathrm{H}), 4.90(\mathrm{~s}, 1 \mathrm{H}), 4.25(\mathrm{~m}, 2 \mathrm{H}), 3.38(\mathrm{~s}, 3 \mathrm{H})$, $2.34(\mathrm{~d}, J=4.9 \mathrm{~Hz}, 3 \mathrm{H}), 1.43(\mathrm{~m}, 3 \mathrm{H}) ;{ }^{13} \mathrm{C} \mathrm{NMR}\left(125 \mathrm{MHz}, \mathrm{CDCl}_{3}\right) \delta$ 168.1, 162.0, 160.5, $146.8,136.9$, 129.9, 129.5, 126.2, 125.7, 123.1, 122.2, 121.0, 120.0, 119.7, 114.2, 111.5, 109.5, 87.8, 83.4, 67.6, 63.4, 61.4, 58.0, 26.1, 14.0; HRMS (CI) calcd for $\mathrm{C}_{25} \mathrm{H}_{26} \mathrm{O}_{5} \mathrm{~N}_{4} \mathrm{Na}$ 485.1801, found 485.1788; $[\alpha]^{23}+63.0\left(c 0.26, \mathrm{CHCl}_{3}\right)$. 


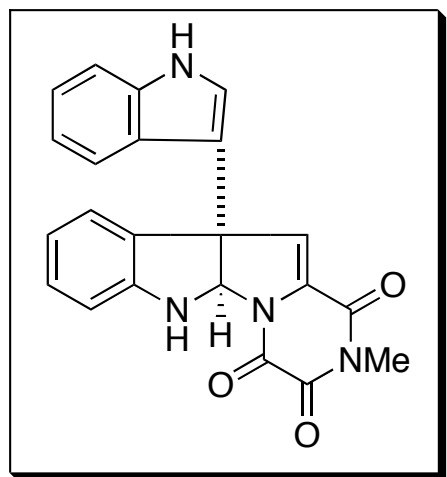

(+)-Gliocladin C (1). Following the general procedure of Mulliez, ${ }^{6} 1,1,1,3,3,3$-hexamethyldisilasane $(1 \mathrm{~mL})$ was added to caroxamide ester ester $15(2.8 \mathrm{mg})$ in sealed tube and the reaction mixture was placed in a $140{ }^{\circ} \mathrm{C}$ oil bath. After $20 \mathrm{~min}$, the tube was removed from the bath, allowed to cool to room temperature, and volatile components were removed under high vacuum. Flash column chromatography (EtOAc : hexane $=1$ : 1) of the crude residue yielded $1.7 \mathrm{mg}(73 \%)$ of $(+)$-gliocladin $\mathrm{C}$ (1) as a yellow powder: IR (film) 3351, 1679, 1470, $1318 \mathrm{~cm}^{-1}$; ${ }^{1} \mathrm{H}$ NMR (600 MHz, Acetone-d ${ }_{6}$ ) $\delta 10.33$ (br s, 1H), 7.43 (br d $J$ $=8.2 \mathrm{~Hz}, 1 \mathrm{H}), 7.33(\mathrm{dd}, J=0.7,8.2 \mathrm{~Hz}, 1 \mathrm{H}), 7.23(\mathrm{~d}, J=2.6$ $\mathrm{Hz}, 1 \mathrm{H}), 7.18$ (br d, $J=7.4 \mathrm{~Hz}, 1 \mathrm{H}), 7.13$ (ddd, $J=8.7,7.5,1.1 \mathrm{~Hz}, 1 \mathrm{H}), 7.11$ (ddd, $J=8.1$, 7.1, $1.0 \mathrm{~Hz}, 1 \mathrm{H}), 6.96(\mathrm{~s}, 1 \mathrm{H}), 6.90(\mathrm{ddd}, J=8.0,7.1,0.8 \mathrm{~Hz}, 1 \mathrm{H}), 6.86$ (br d, $J=7.9 \mathrm{~Hz}$, $1 \mathrm{H}), 6.72$ (ddd, $J=7.4,7.3,1.0 \mathrm{~Hz}, 1 \mathrm{H}), 6.61$ (br d, $J=1.9 \mathrm{~Hz}, 1 \mathrm{H}), 6.24$ (d, $J=2.6 \mathrm{~Hz}$, $1 \mathrm{H}), 3.26(\mathrm{~s}, 3 \mathrm{H}) ;{ }^{13} \mathrm{C}$ NMR $\left(125 \mathrm{MHz}\right.$, acetone-d $\left.{ }_{6}\right) \delta 158.62,158.01,150.70,149.93$, $138.47,133.08,131.11,129.68,126.94,126.29,125.47,123.70,122.76,120.13,120.11$, 119.65, 116.66, 112.63, 110.58, 84.69, 60.97, 27.09; HRMS (CI) calcd for $\mathrm{C}_{22} \mathrm{H}_{16} \mathrm{~N}_{4} \mathrm{O}_{3} \mathrm{Na}$ $407.1120(\mathrm{M}+\mathrm{Na})$, found 407.1118; $[\alpha]^{27}{ }_{\mathrm{D}}+116.4\left(c 0.02, \mathrm{CHCl}_{3}\right)$, reported $^{7}[\alpha]_{\mathrm{D}}+131.4(c$ $\left.0.07, \mathrm{CHCl}_{3}\right)$.

\footnotetext{
${ }^{6}$ Mulliez, M.; Royer, J. Tetrahedron 1984, 40, 5143-5151.

7 Usami, Y.; Yamaguchi, J.; Numata, A. Heterocycles 2004, 63, 1123-1129.
} 


\section{B. Tabulated ${ }^{1} \mathrm{H}$ and ${ }^{13} \mathrm{C}$ NMR spectra of natural and synthetic (+)-gliocladin $\mathrm{C}$}

\section{LITERATURE (500 MHz, acetone- $\mathrm{d}_{6}$ )}

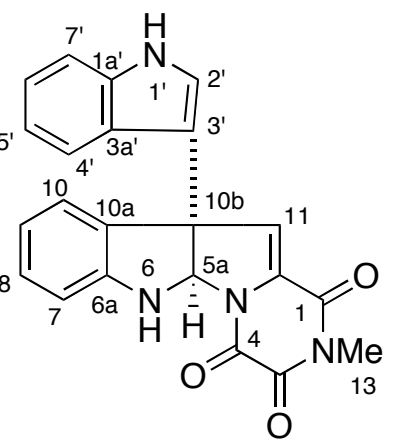

SYNTHETIC (600 MHz, acetone- $d_{6}$ )

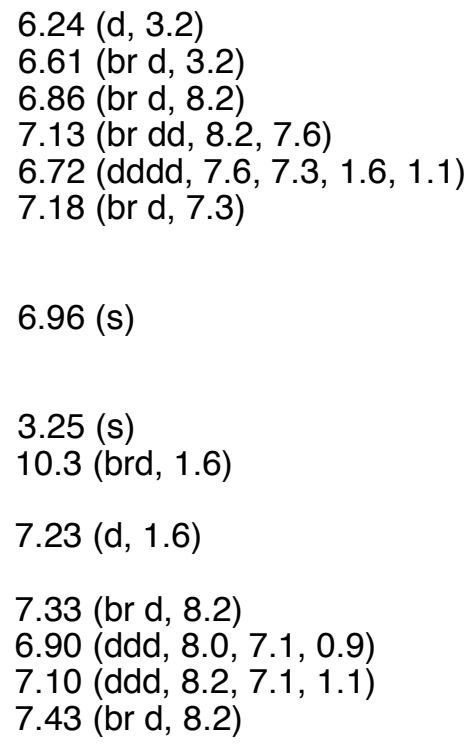

Source : Heterocycles 2004, 63, 1123 
LITERATURE (125 MHz, acetone- $d_{6}$ )

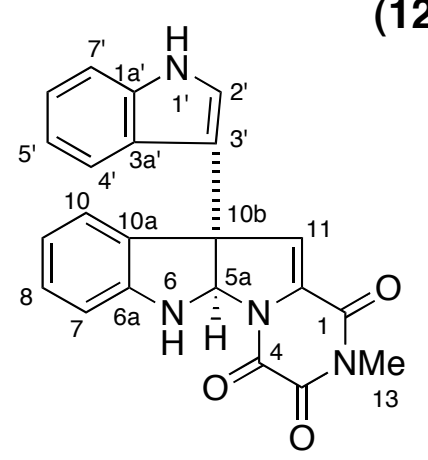

SYNTHETIC

(125 MHz, acetone- $d_{6}$ )

$\begin{array}{ll}1 & 158.62 \\ 3 & 158.01 \\ 4 & 150.71 \\ 5 a & 84.72 \\ 6 a & 149.94 \\ 7 & 110.59 \\ 8 & 129.68 \\ 9 & 119.66 \\ 10 & 125.47 \\ 10 a & 131.12 \\ 10 b & 61.00 \\ 11 a & 126.97 \\ 11 b & \\ 12 & 133.08 \\ 13 & 27.10 \\ 1^{\prime} & \\ 1 a^{\prime} & 138.49 \\ 2^{\prime} & 123.71 \\ 3^{\prime} & 122.75 \\ 3 a^{\prime} & 123.55 \\ 4^{\prime} & 119.71 \\ 5^{\prime} & 119.71 \\ 6^{\prime} & 120.12 \\ 7^{\prime} & 112.64\end{array}$

$\begin{array}{ll}1 & 158.62 \\ 3 & 158.01 \\ 4 & 150.70 \\ 5 a & 84.69 \\ 6 a & 149.93 \\ 7 & 110.58 \\ 8 & 129.68 \\ 9 & 119.65 \\ 10 & 125.47 \\ 10 a & 131.11 \\ 10 b & 60.97 \\ 11 a & 126.94 \\ 11 b & \\ 12 & 133.08 \\ 13 & 27.09 \\ 1^{\prime} & \\ 1 a^{\prime} & 138.47 \\ 2^{\prime} & 123.70 \\ 3^{\prime} & \mathbf{1 1 6 . 6 6} \\ 3^{\prime} & \mathbf{1 2 6 . 2 9} \\ 4^{\prime} & \mathbf{1 2 0 . 1 1} \\ 5^{\prime} & \mathbf{1 2 0 . 1 3} \\ 6^{\prime} & \mathbf{1 2 2 . 7 6} \\ 7^{\prime} & 112.63\end{array}$

confirmed by HMQC, HMBC 

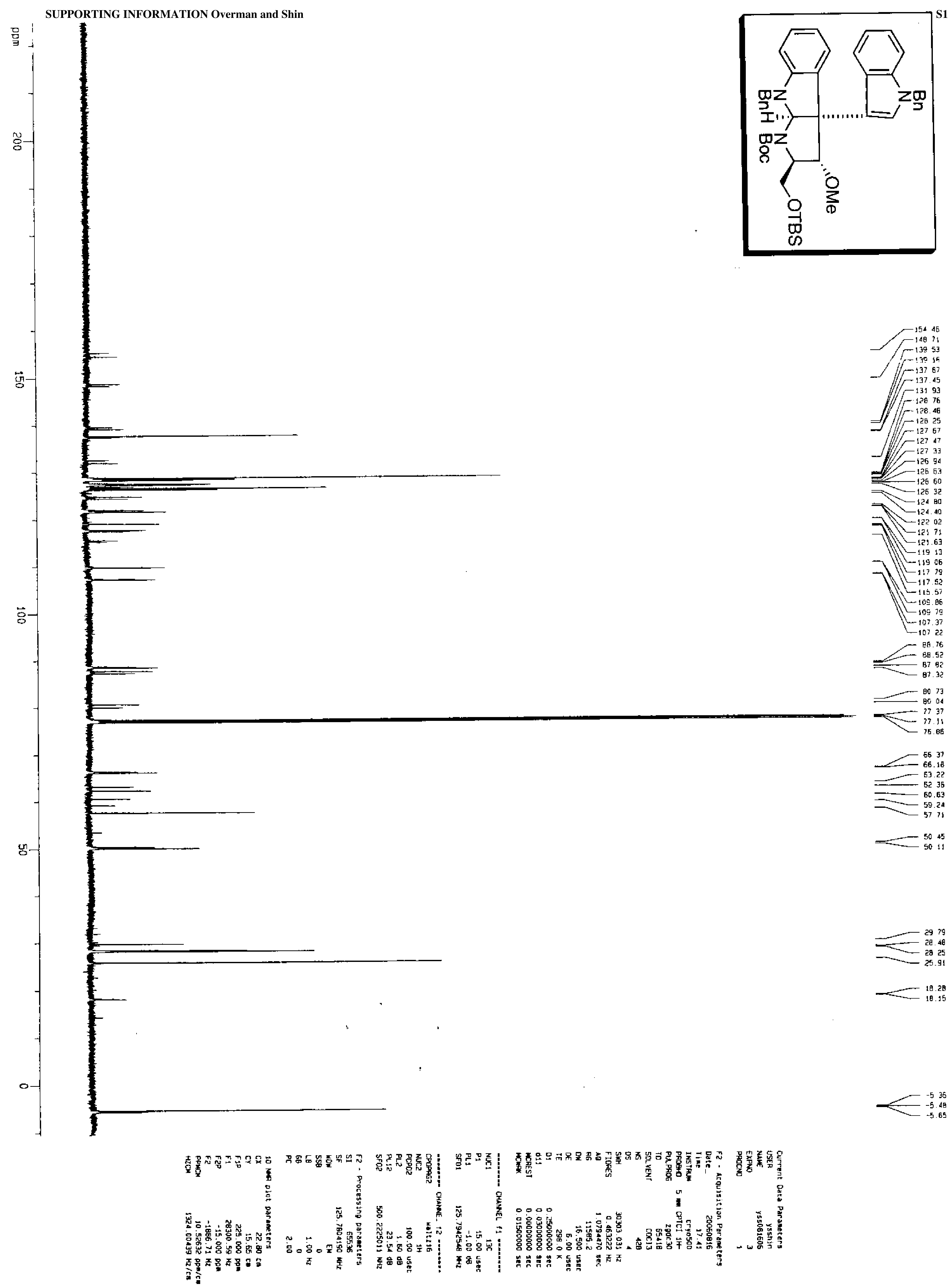


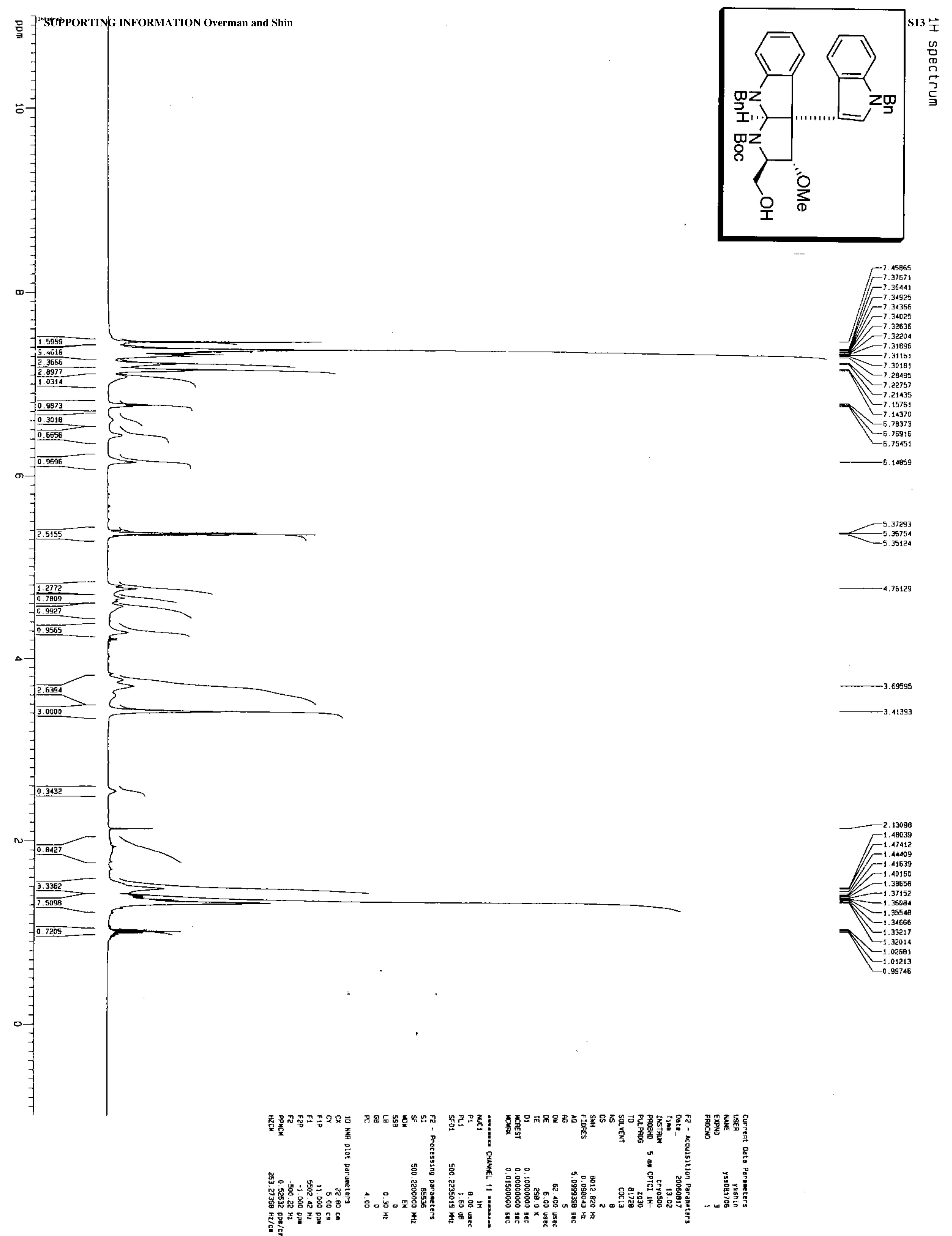




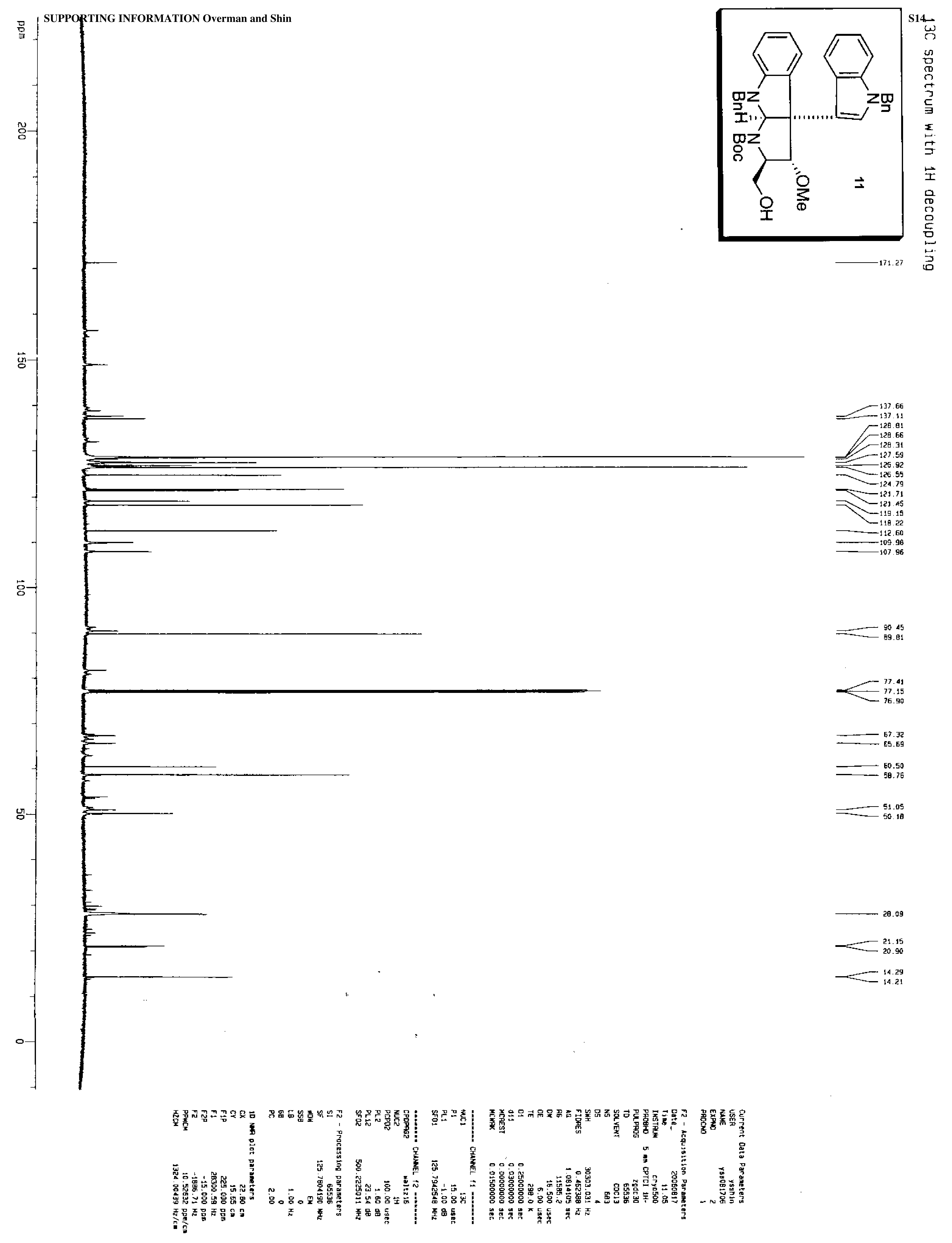




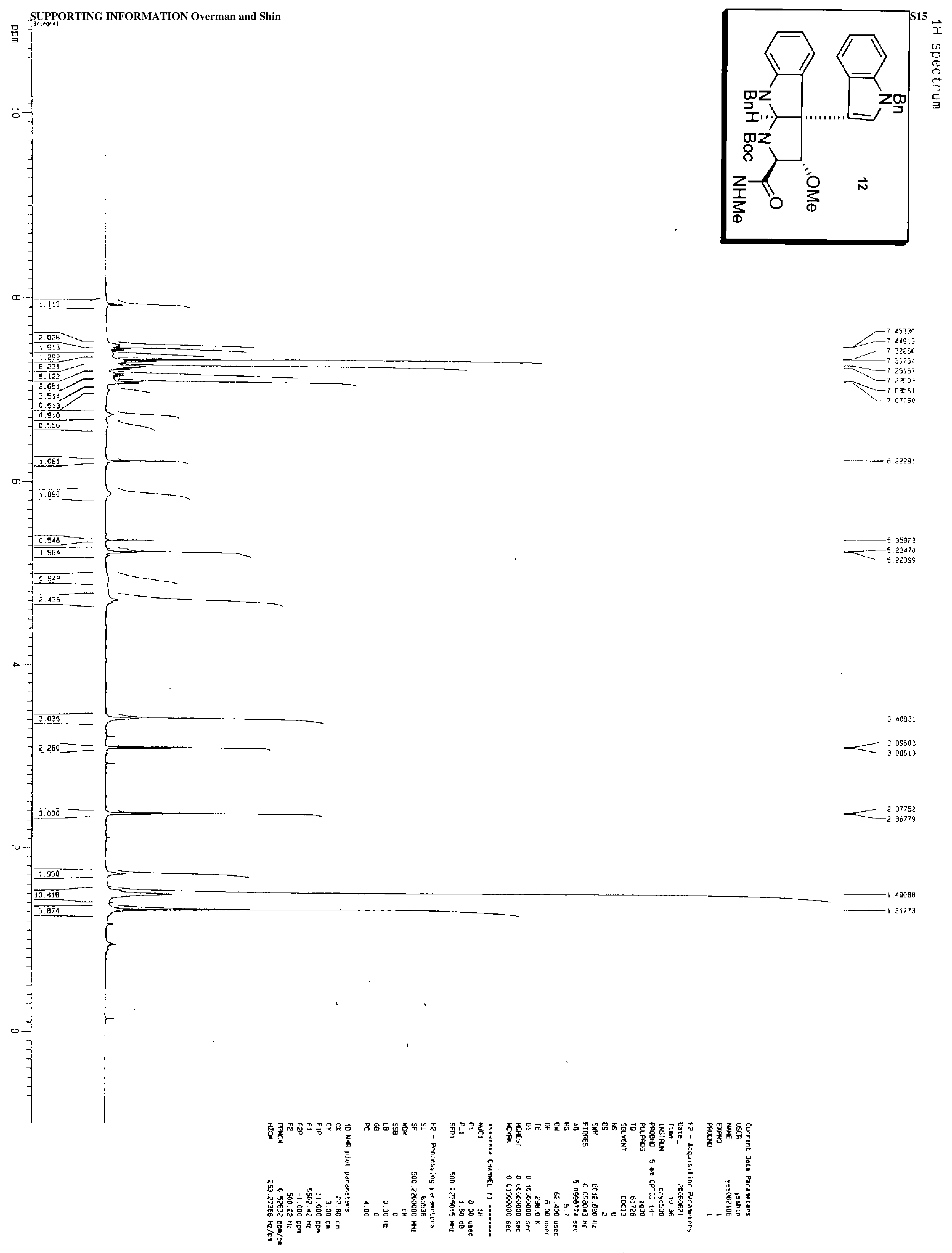



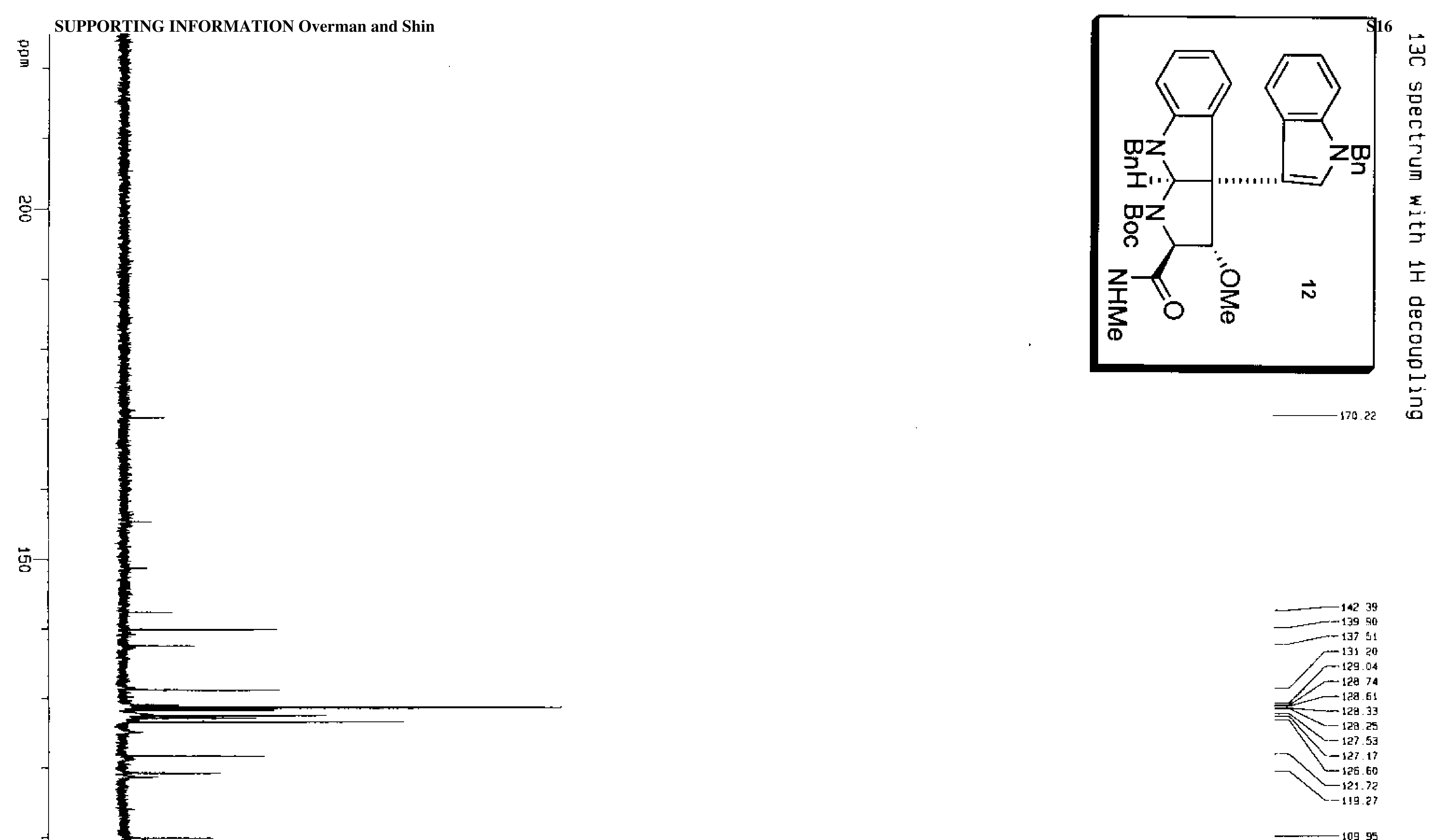

$\overrightarrow{8}$

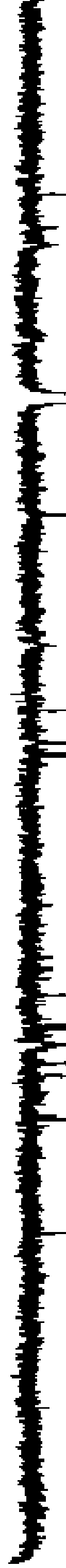

$-9250$

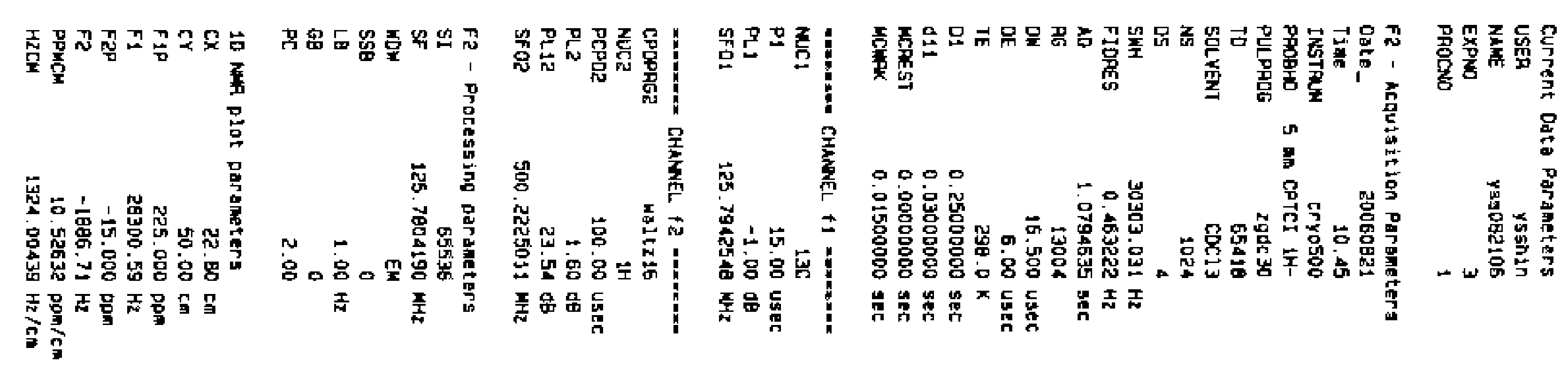




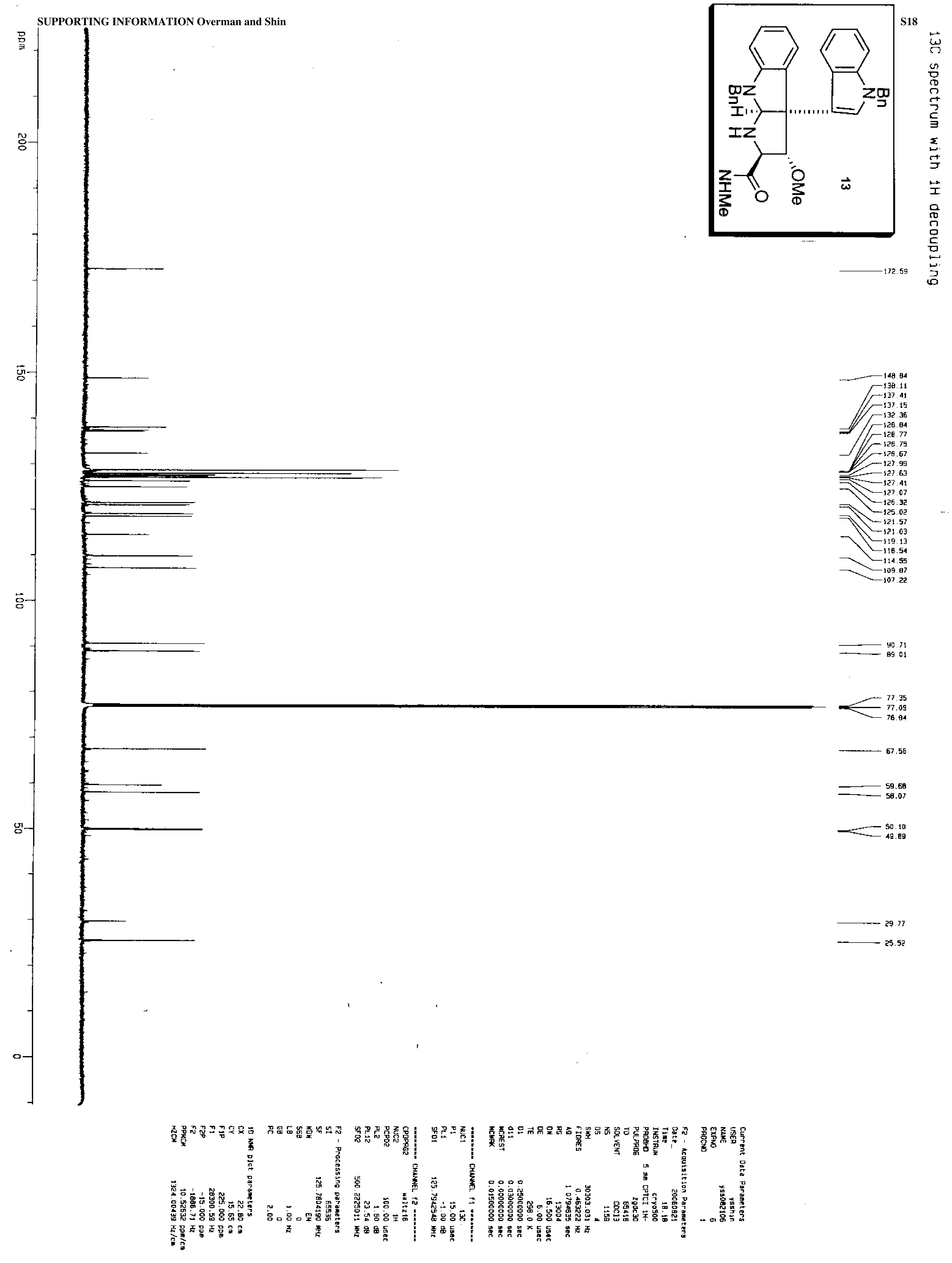



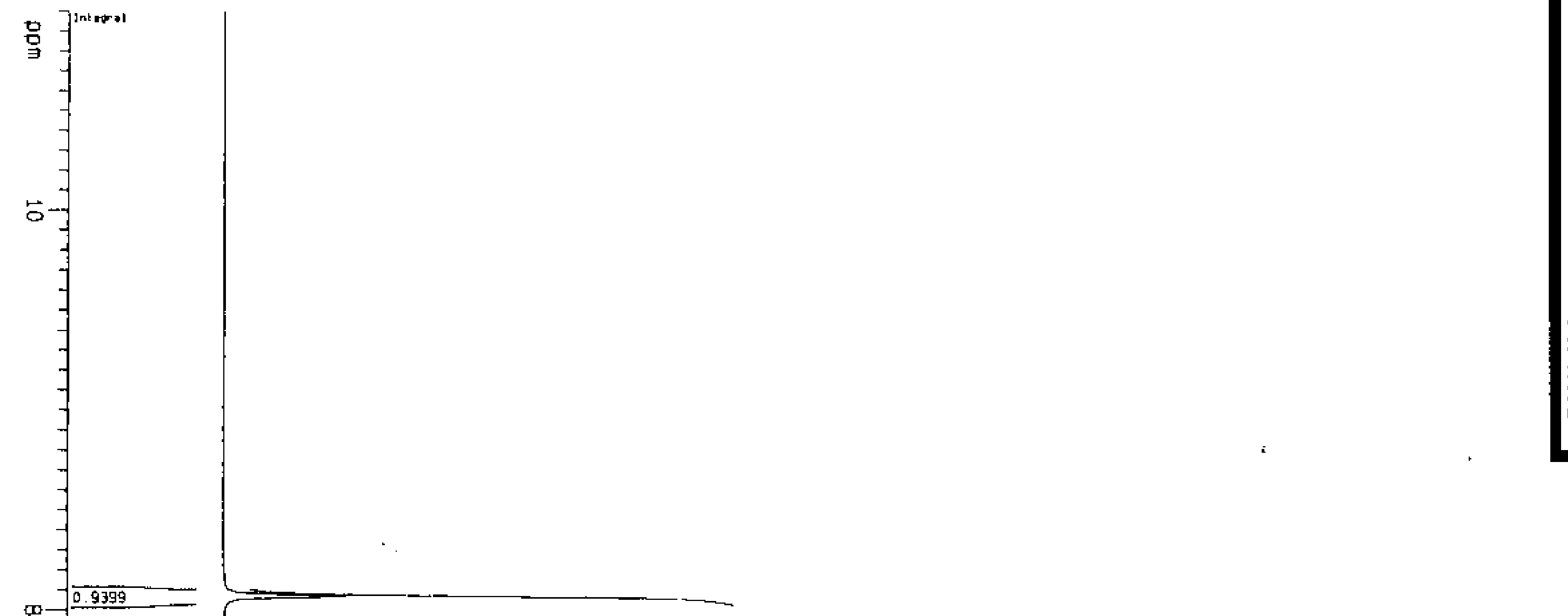

$\infty$
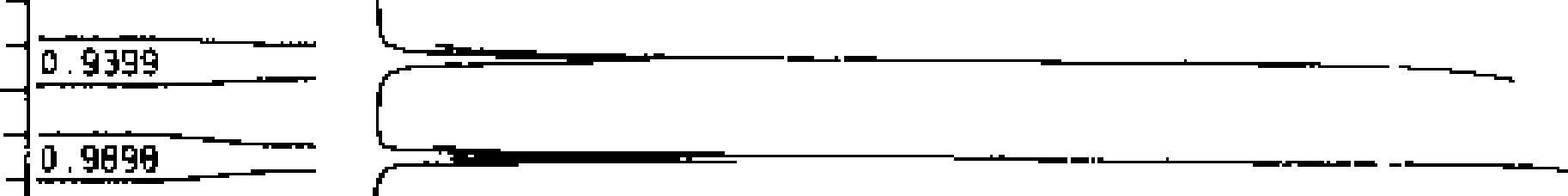

$={ }^{-0.06650}$

F 39758

$F_{-7}^{730146}$

$F^{2}{ }^{27902}$

$F_{-7.13211}^{7.123}$

$\sum_{-7}^{7} 1.9933$

$=7,10152$

$-7.03092$

$=\sqrt{-1}-701770$

-709770
-700252
-6.99543

-5.92057
-6.71675

$\mathrm{U}_{6}^{-6.70183}$

$-8.63160$

$\sigma$

$\sqrt{0.3531}$

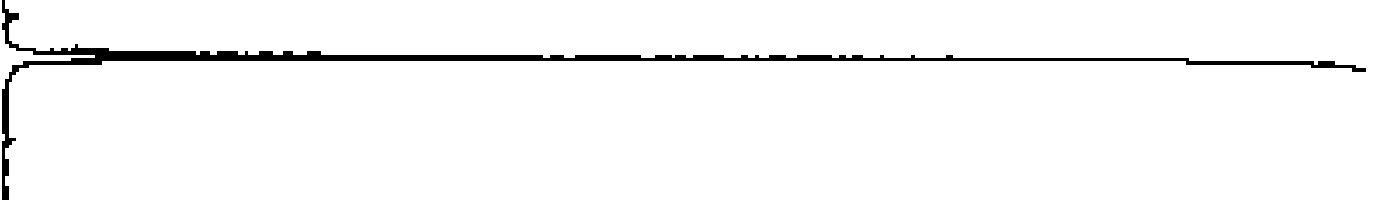

-..-56. 61795

5.31164

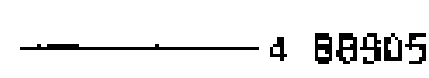

$09716=$

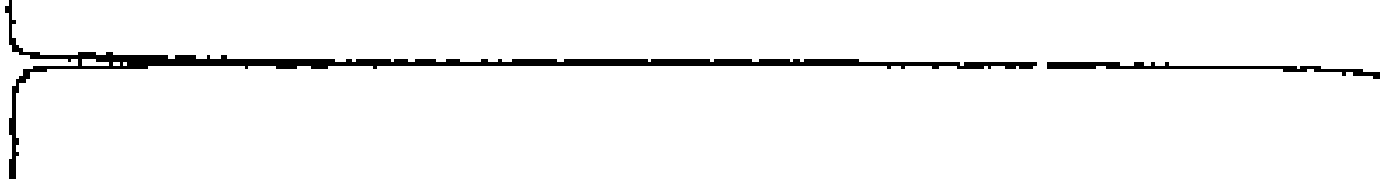

-

905

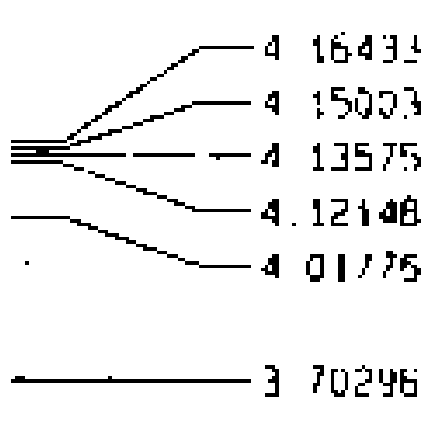

16431 .5003

12140

70246

$-3.36539$

3.0000

$\sqrt{0.6287}=$

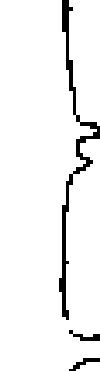

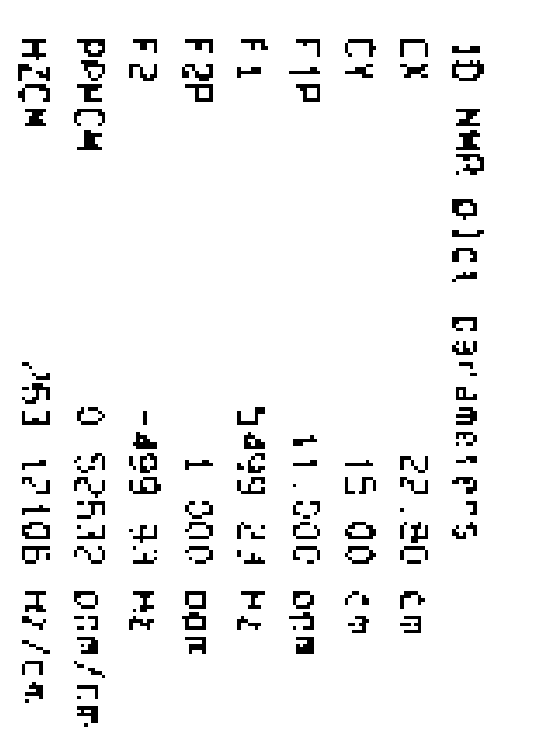

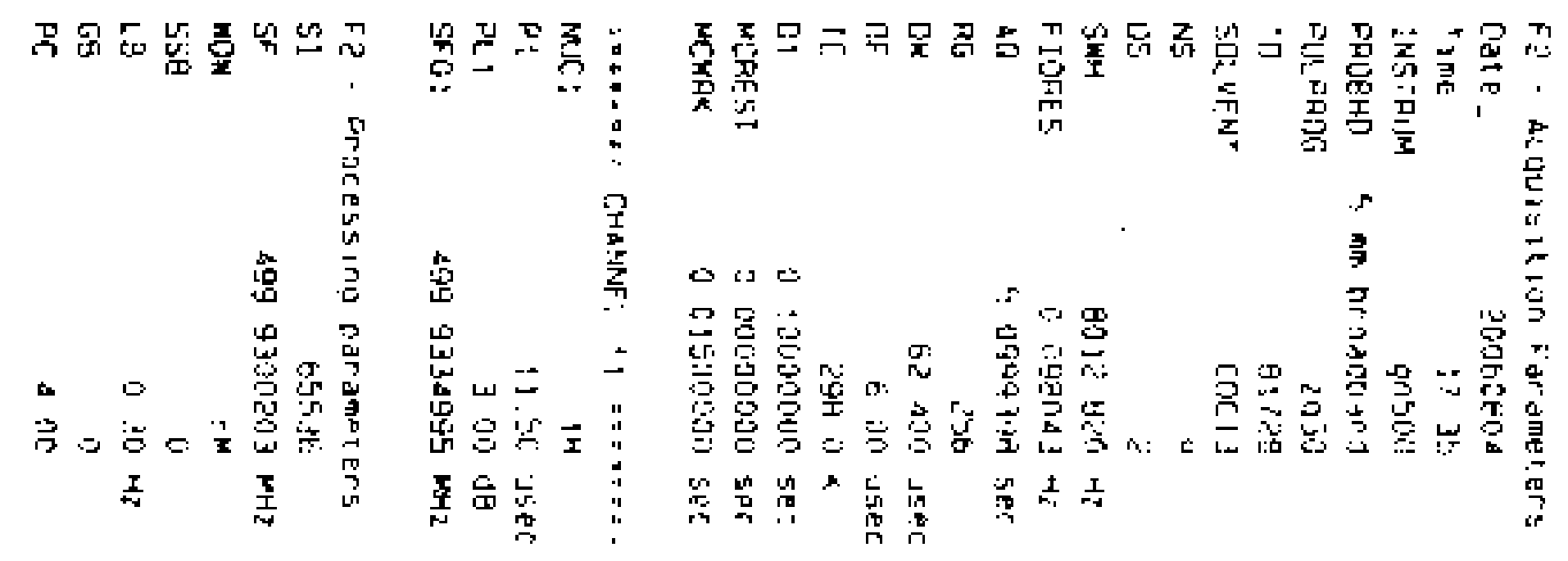

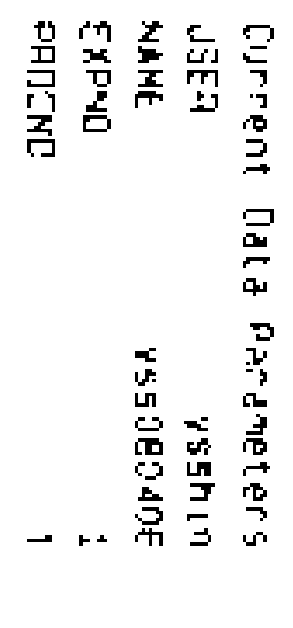




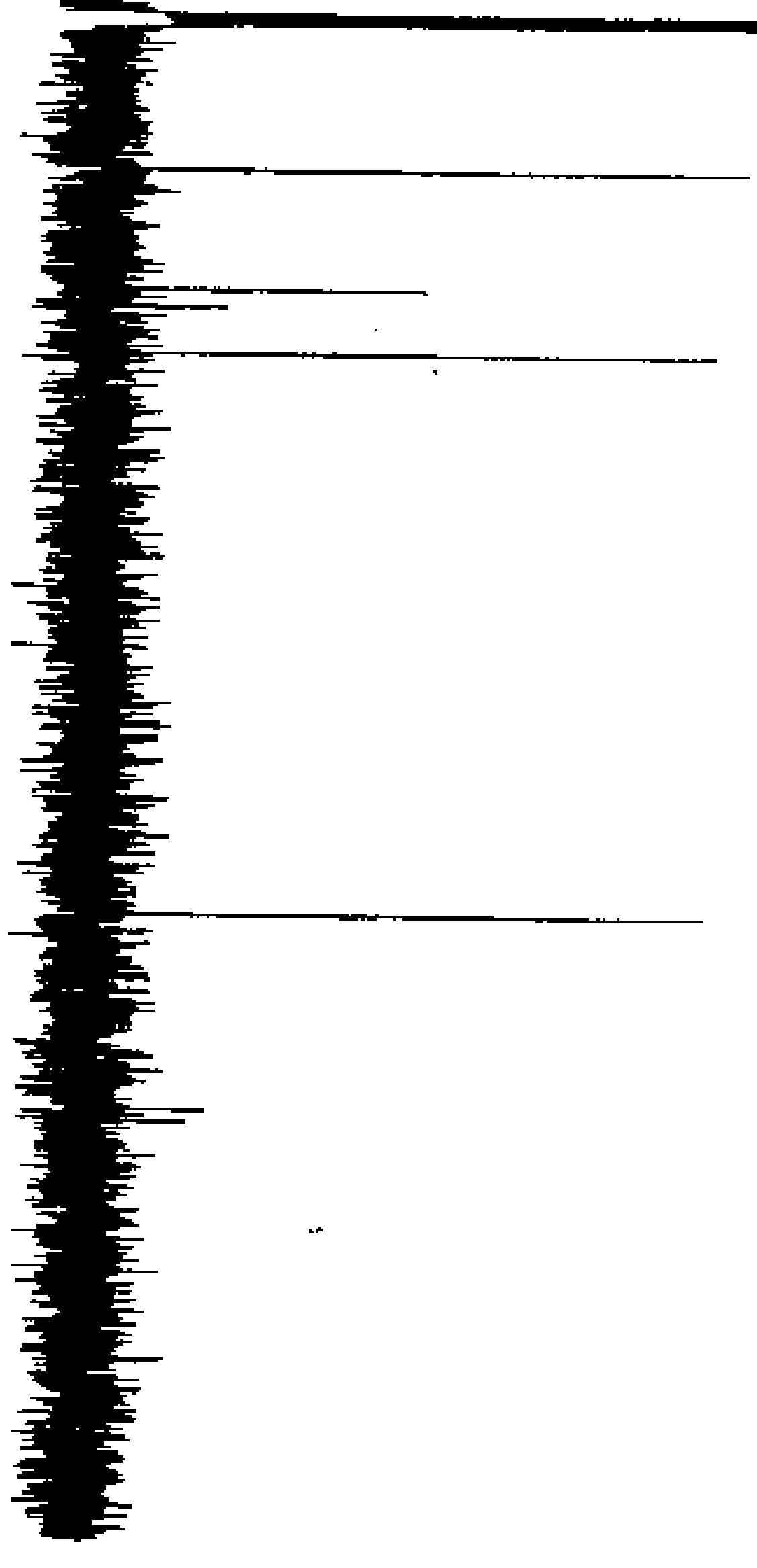

$\underbrace{77.33}$ 

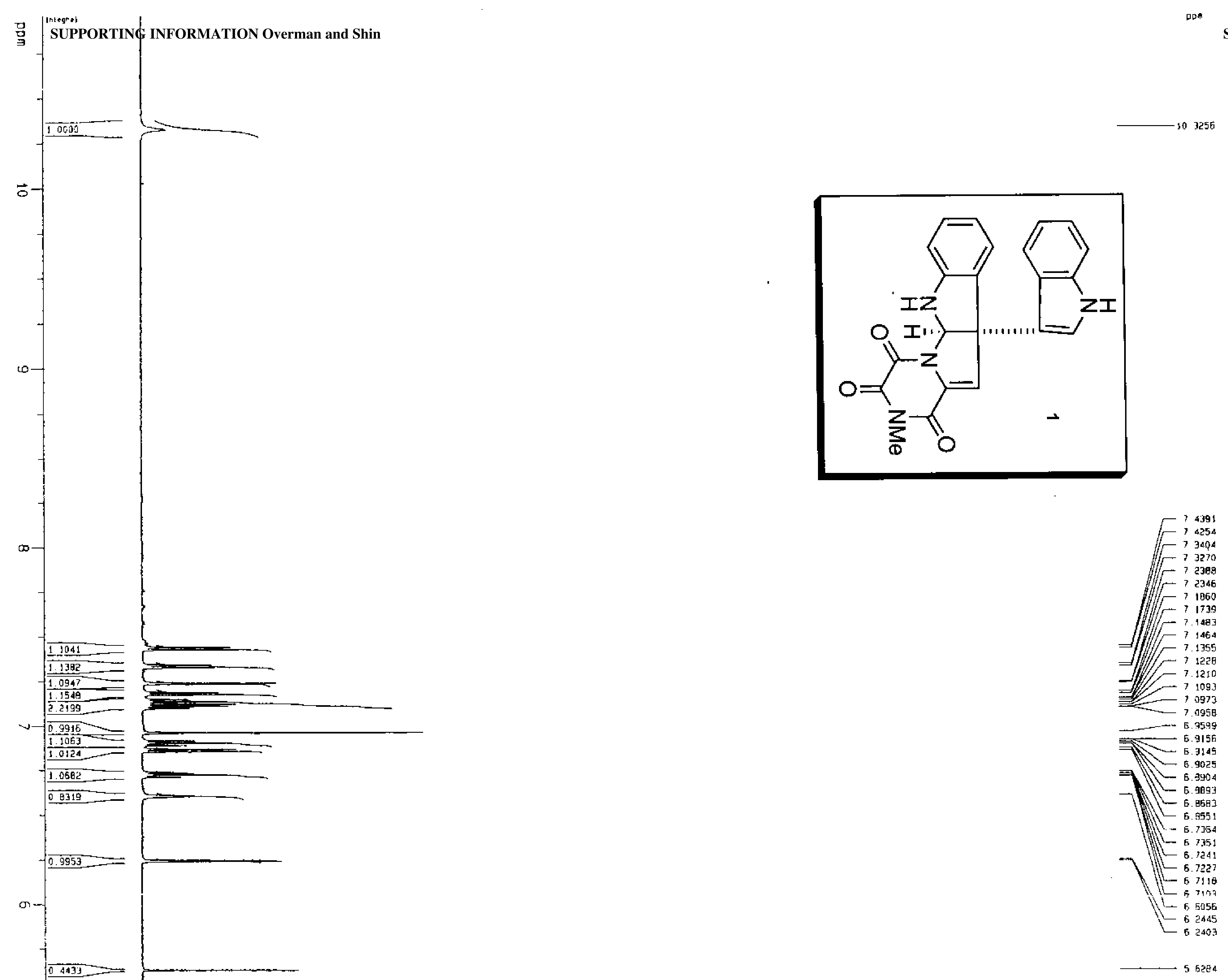
SUPPORTING INFORMATION Overman and Shin

\section{뭅}

\section{$:$}

$\stackrel{20}{3}$

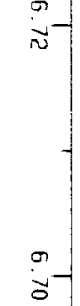

\section{$:-$}


SUPPORTING INFORMATION Overman and Shin

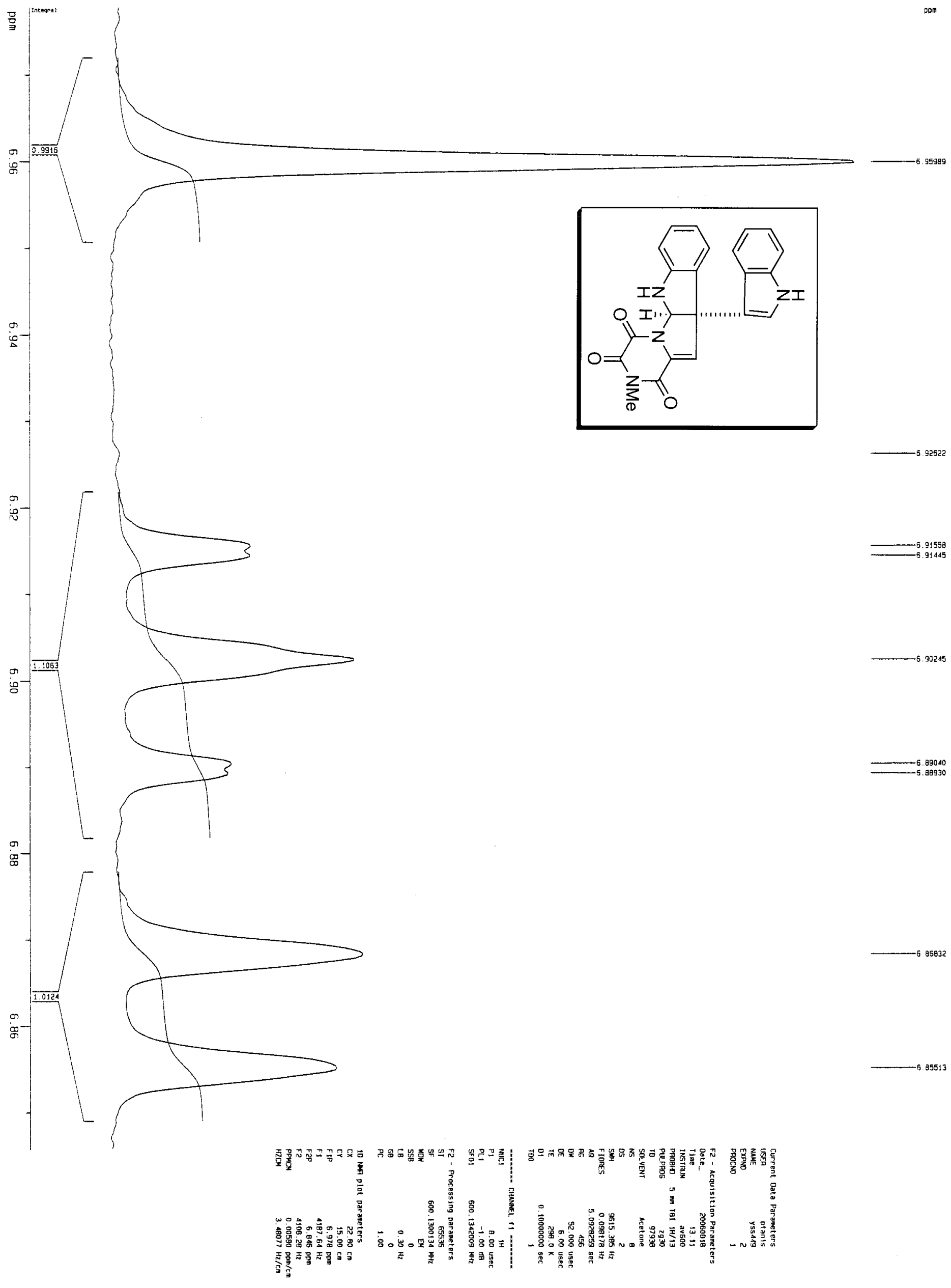




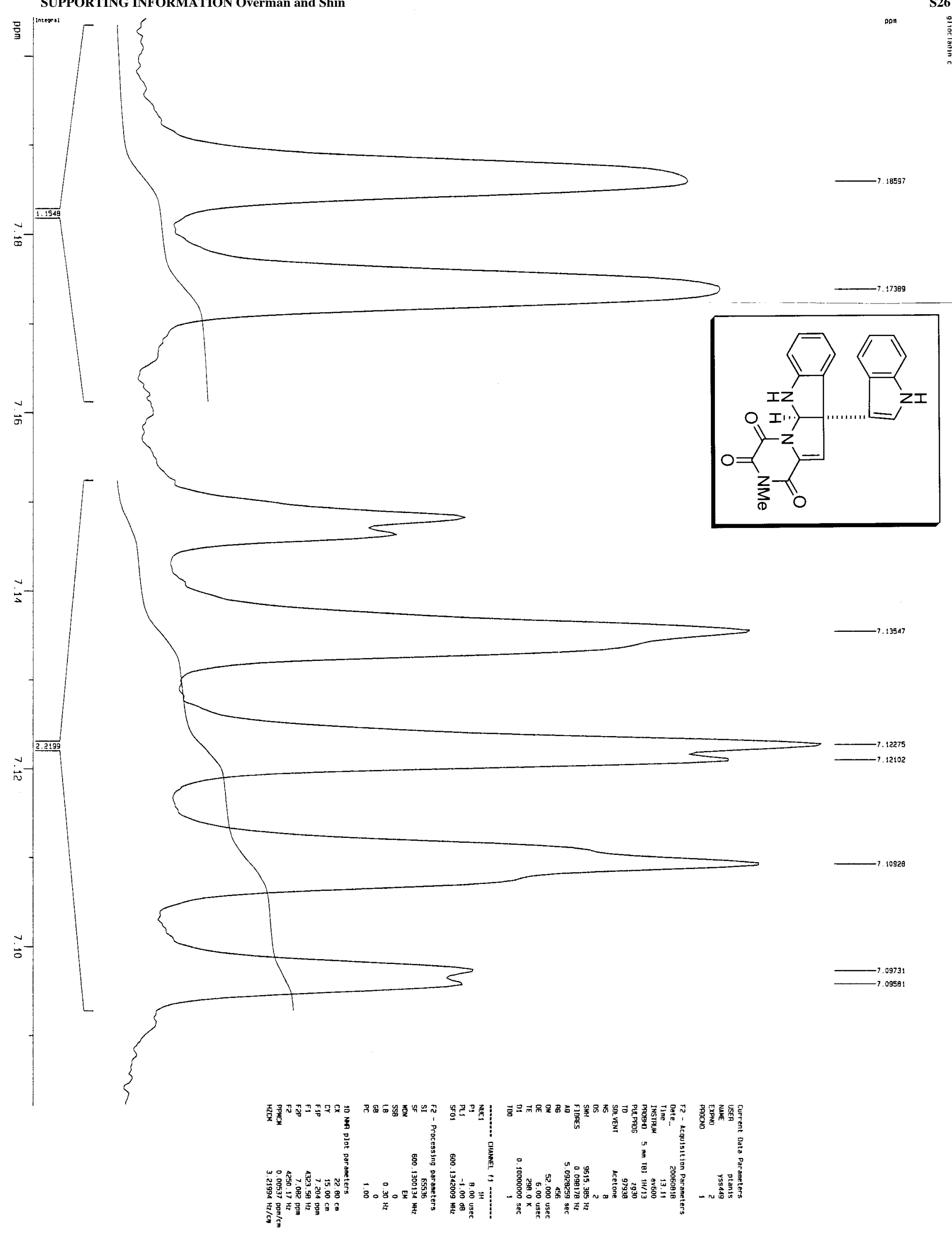




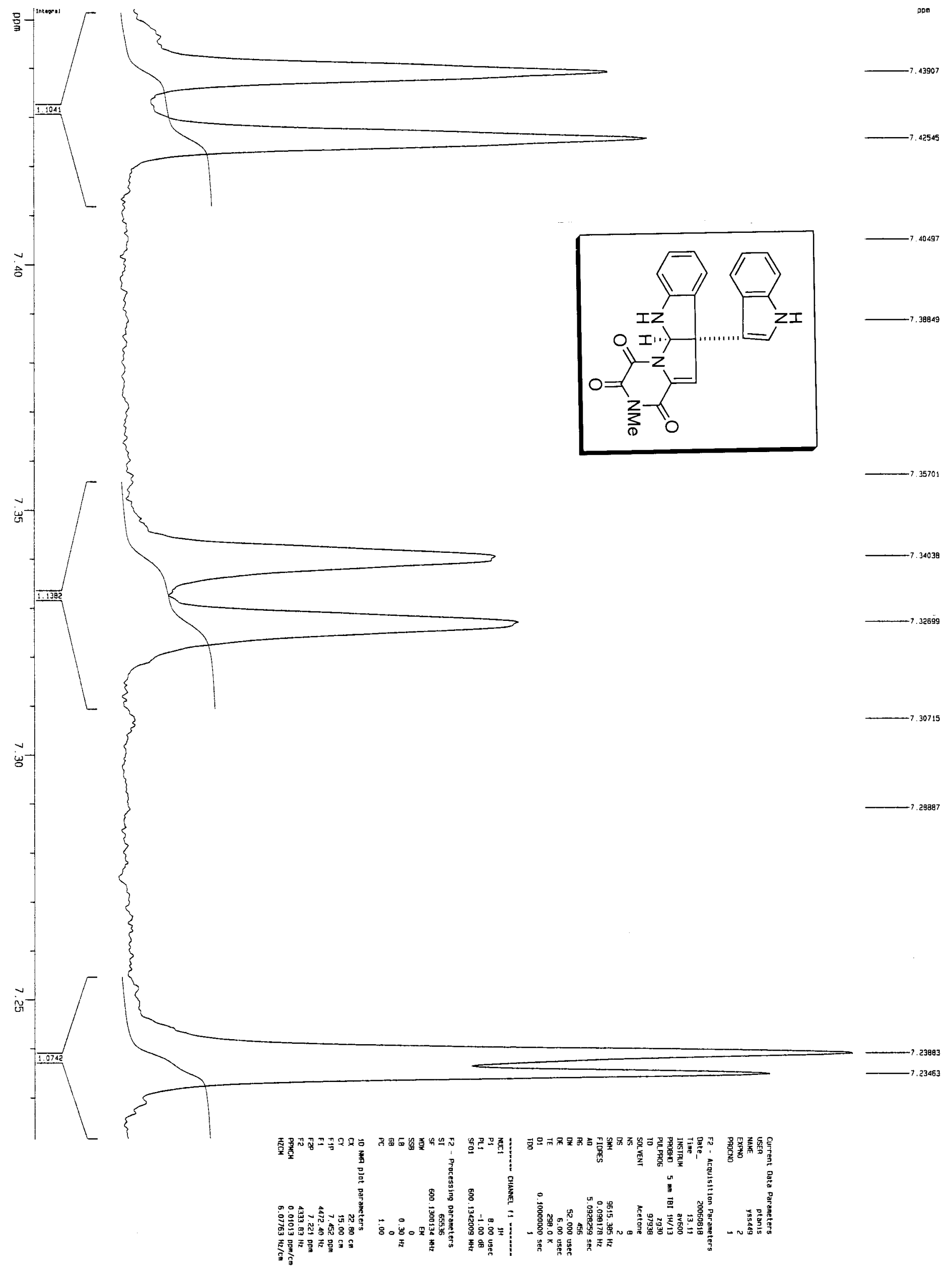



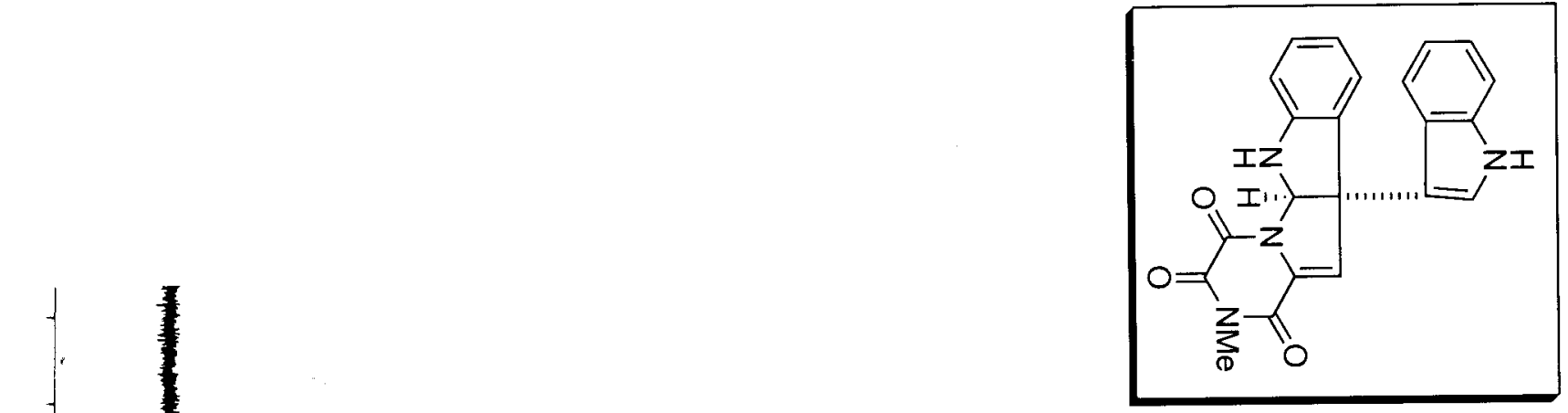
SUPPORTING INFORMATION Overman and Shin
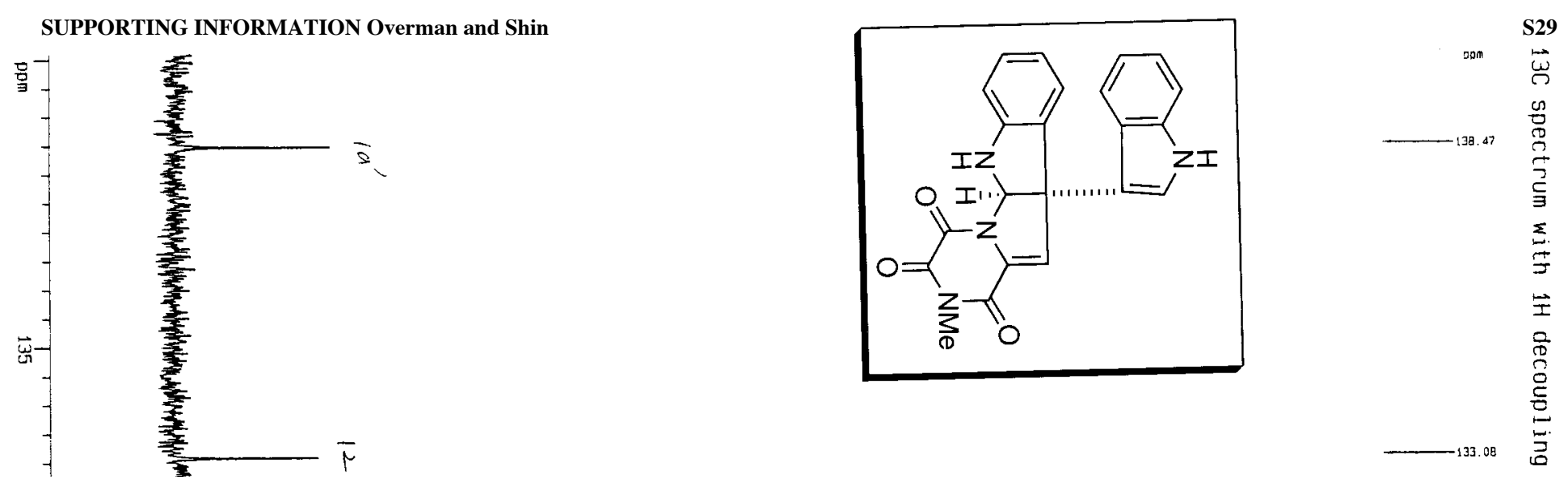

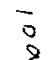

$\longrightarrow$ - 131.11

$\infty$

$-129.58$

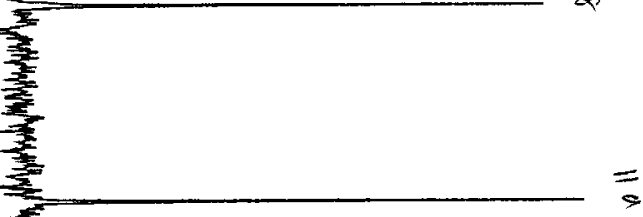

$\frac{1}{5}$

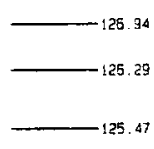

Un-

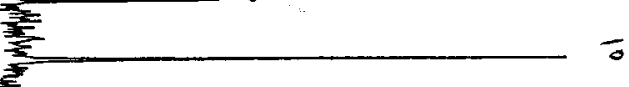

$-123.70$

$\sigma$

$\longrightarrow{ }_{120.11}^{120} 13$

P
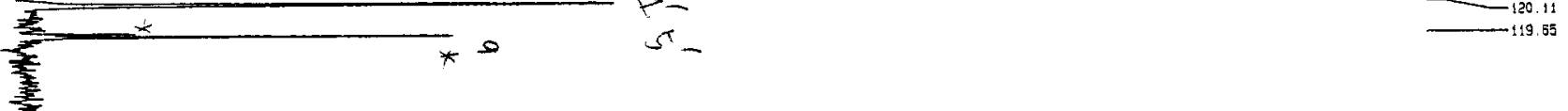

$-146.66$

亦旁

ज-

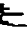

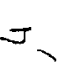

$\longrightarrow$-112.53

${ }_{-110.58}^{110.62}$

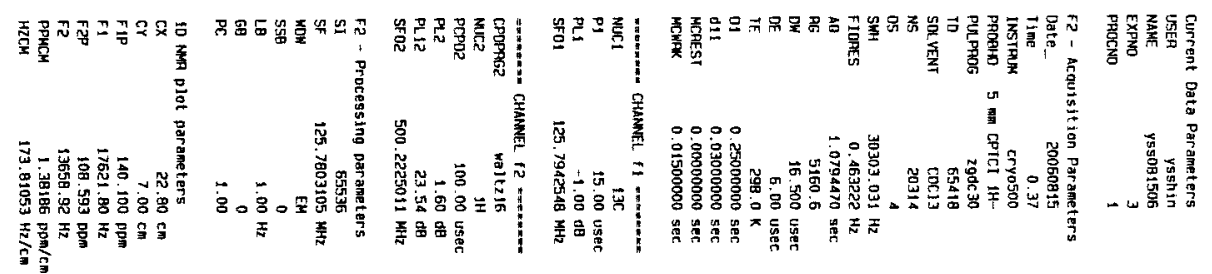


SUPPORTING INFORMATION Overman and Shin

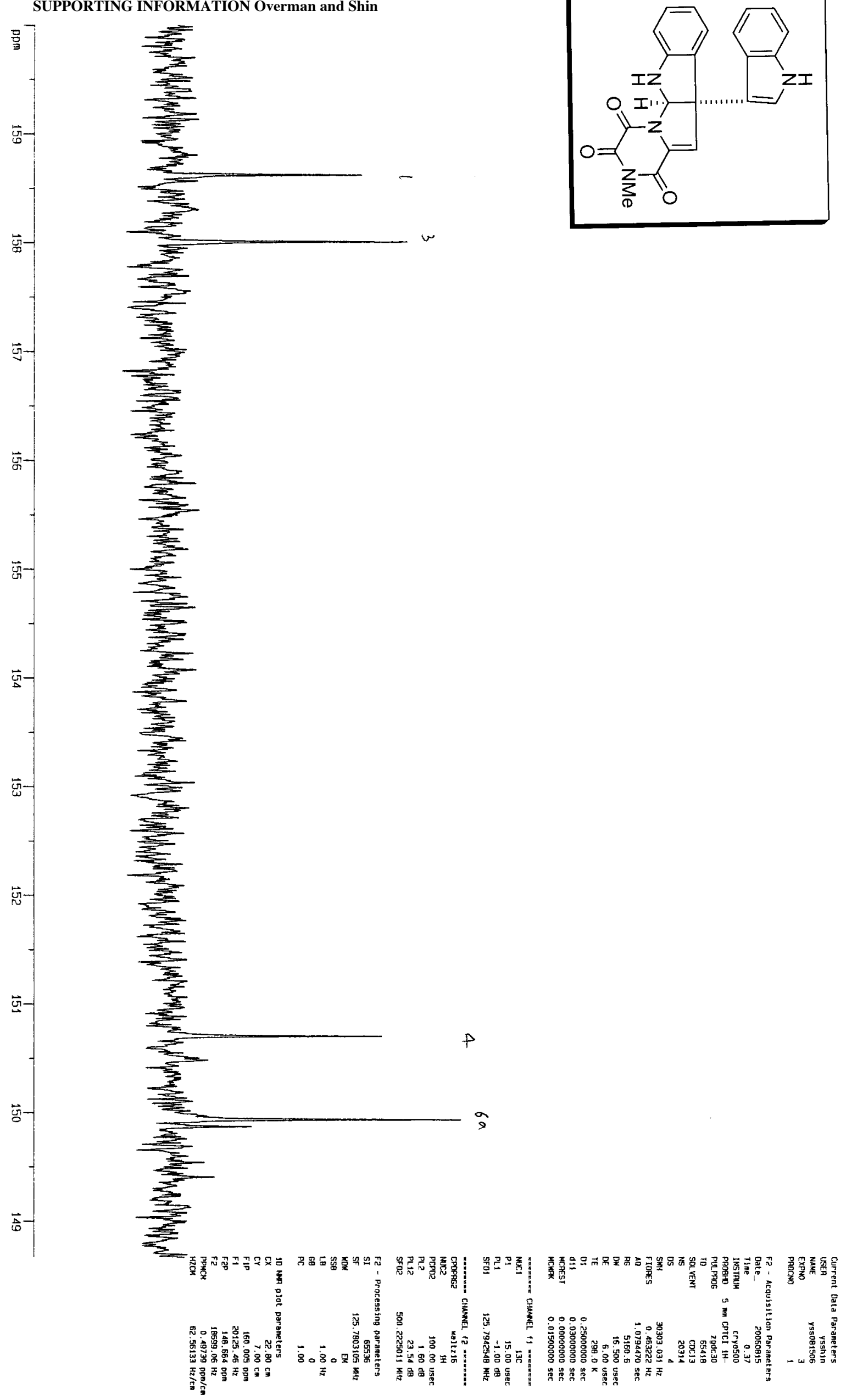



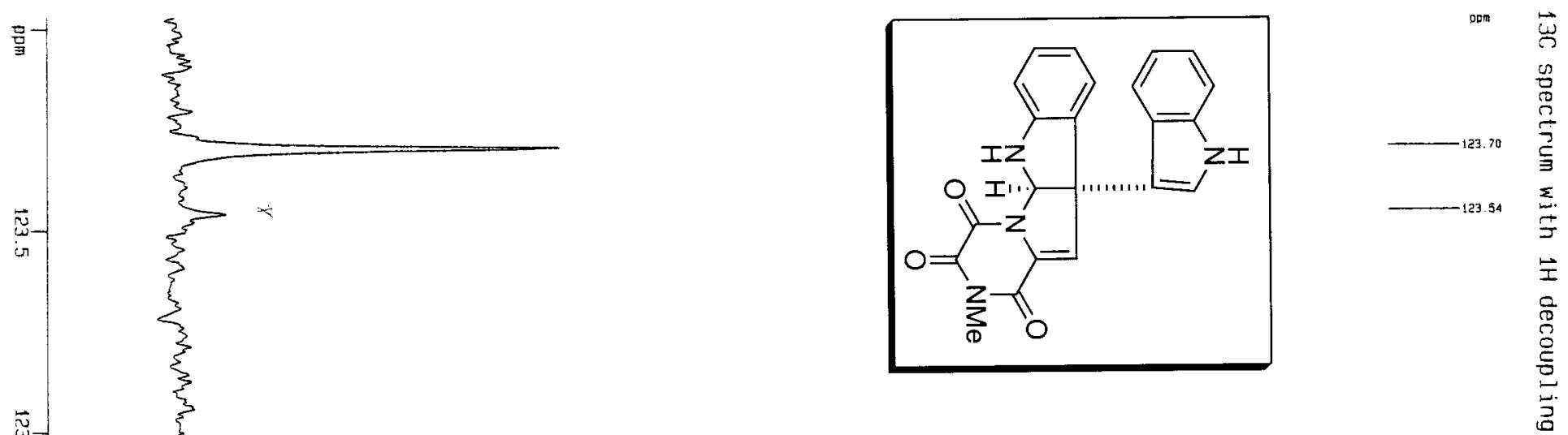

$\vec{\omega}$
0
0

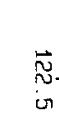

$\vec{N}$

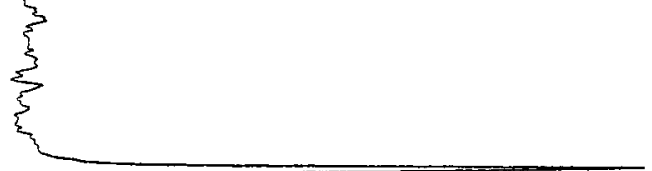

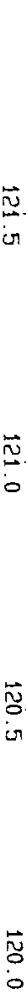

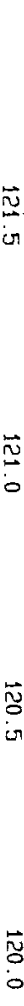

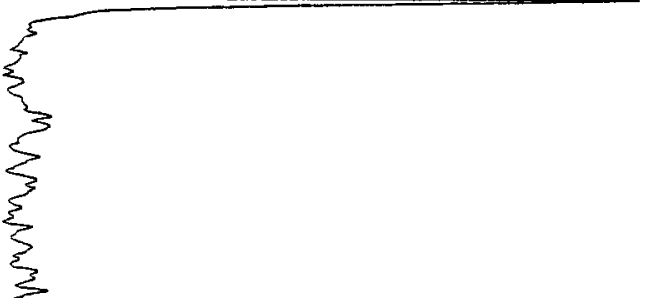

$\stackrel{\sim}{0}$

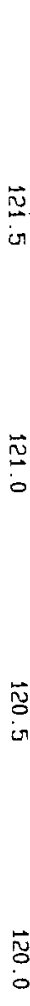

응-

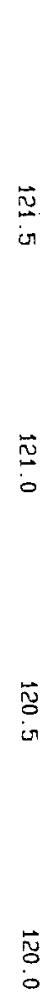

:

\{

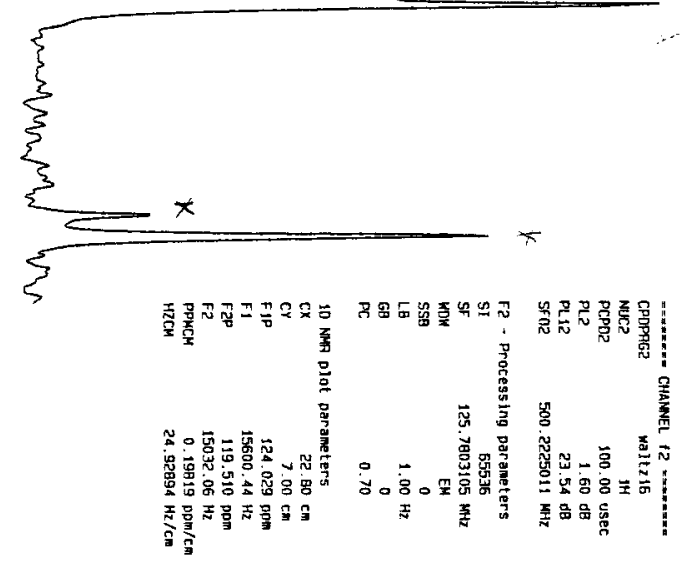

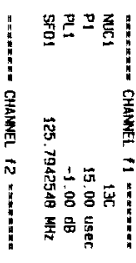

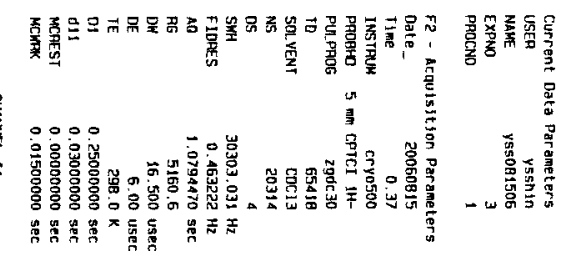



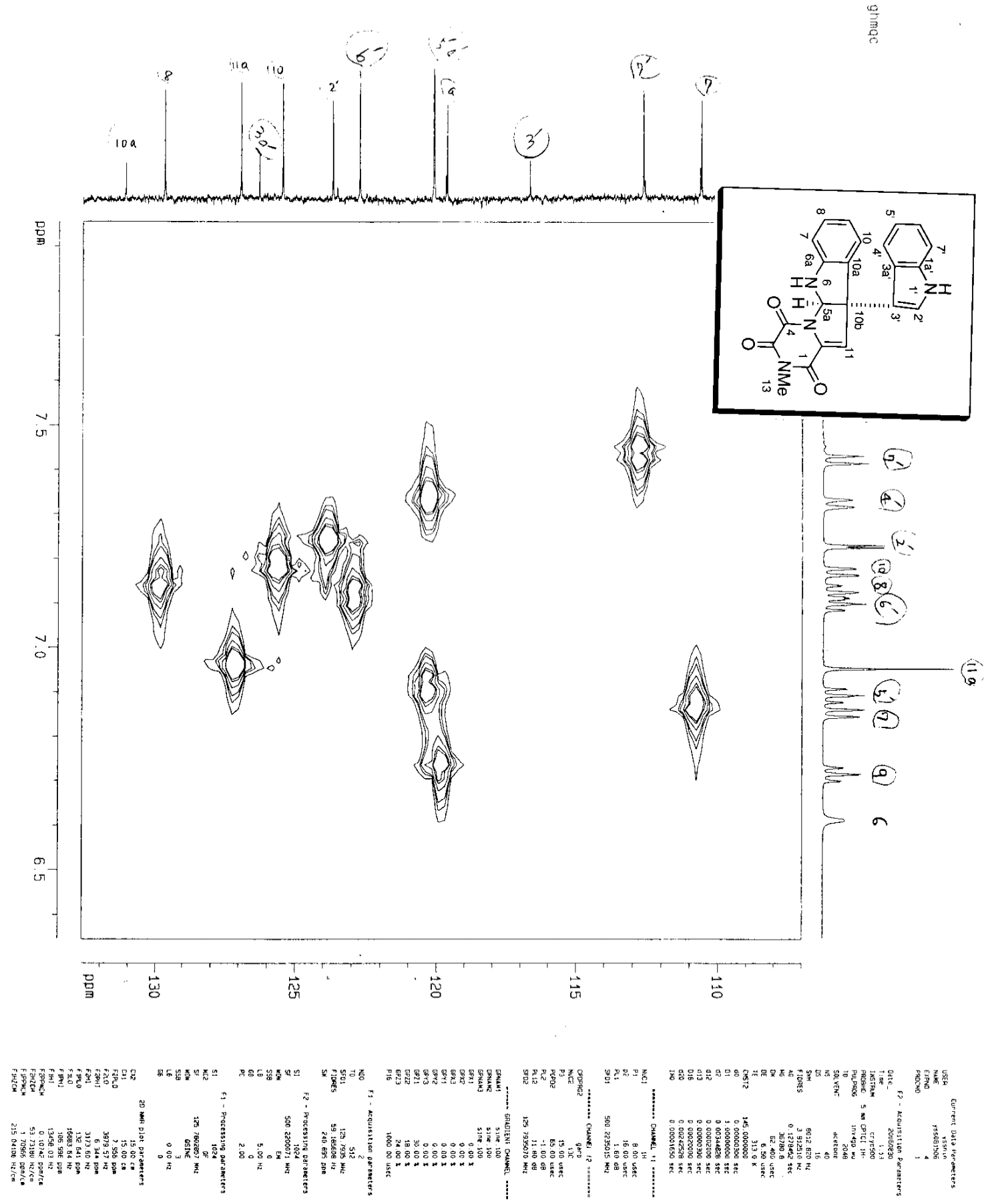


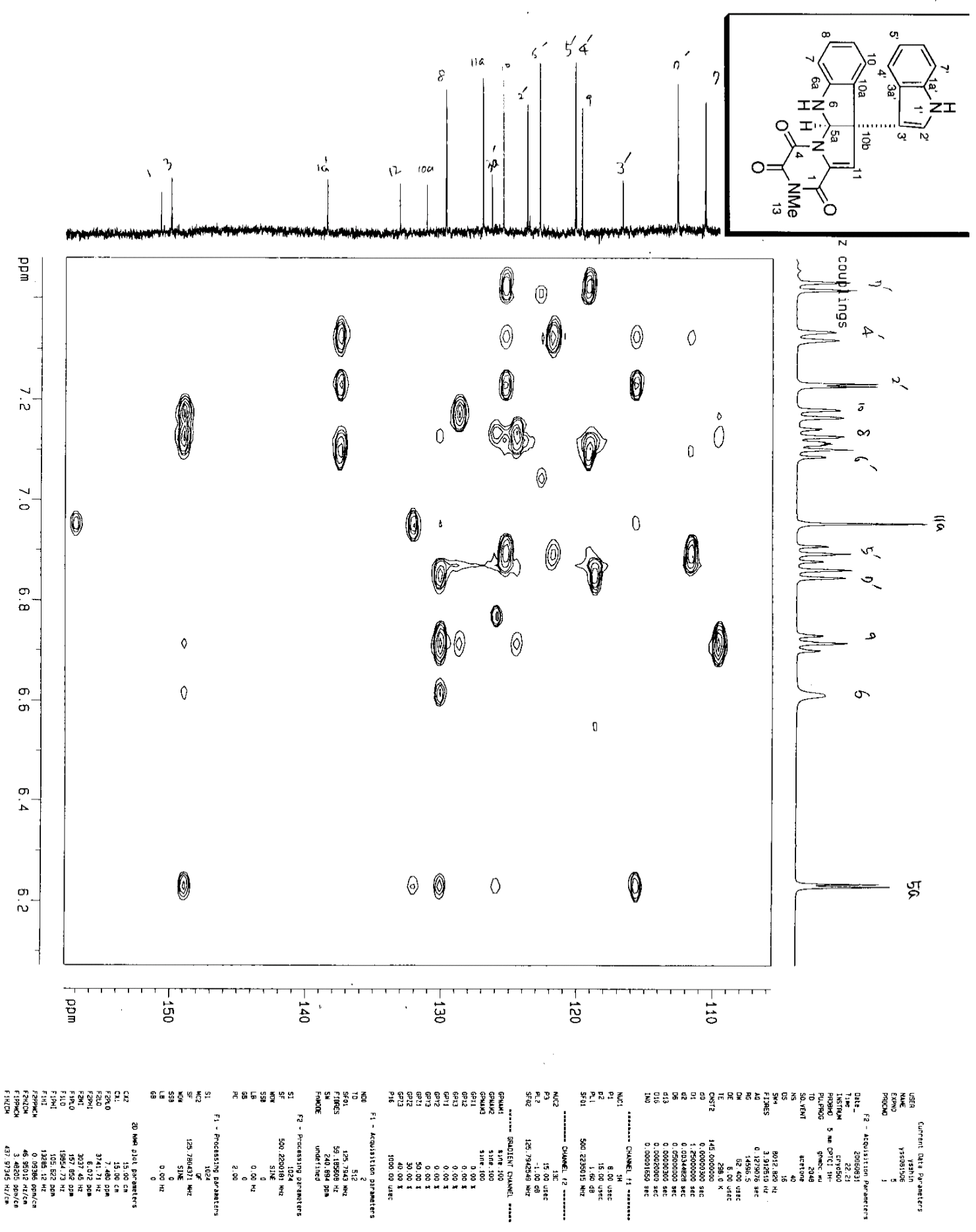




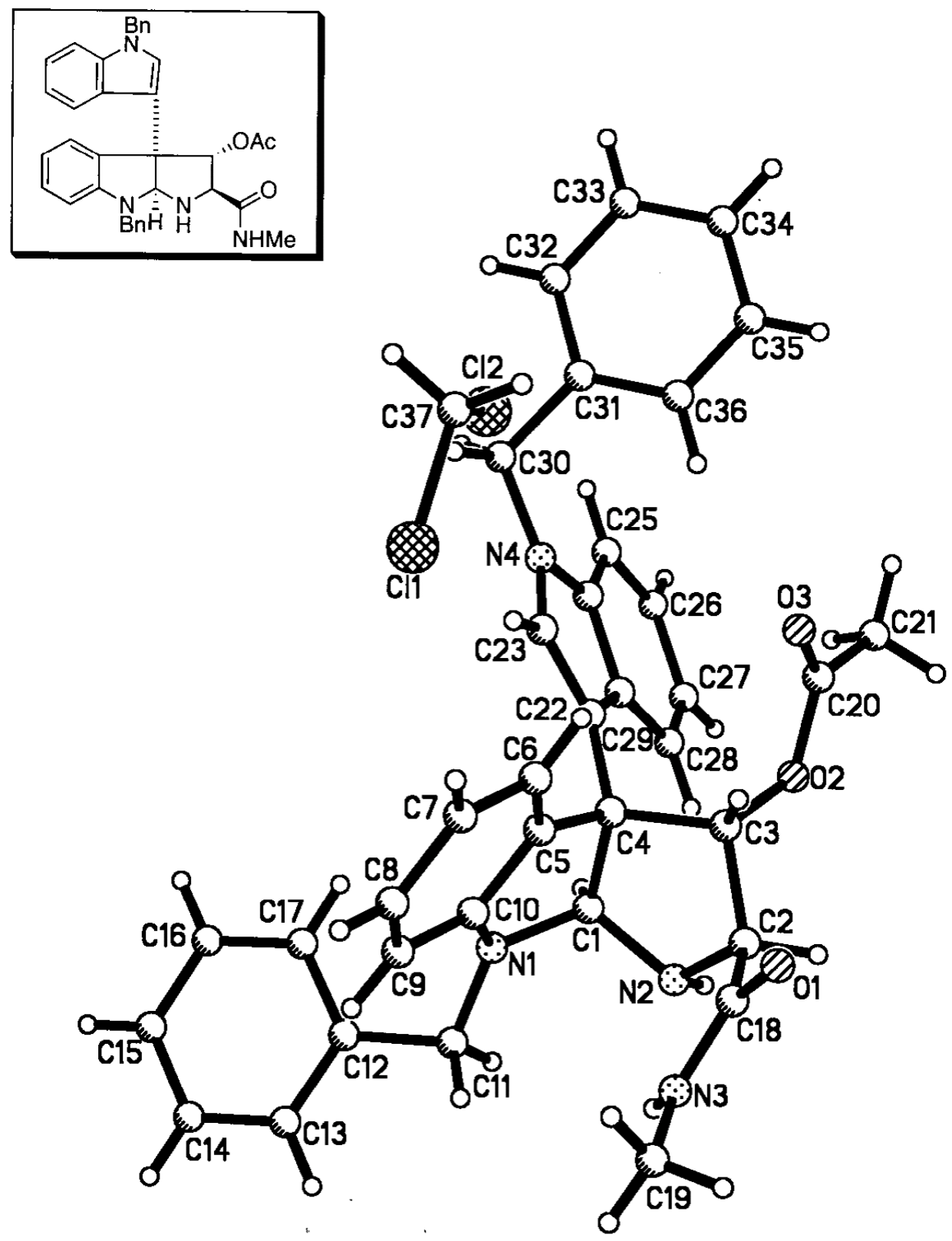

